\title{
Isolation from Cochlea of a Novel Human Intronless Gene with Predominant Fetal Expression
}

\author{
Barbara L. Resendes, ${ }^{1,4}$ Sharon F. Kuo, ${ }^{1,3}$ Nahid G. Robertson, ${ }^{1}$ Anne B. S. Giersch, ${ }^{2,4}$ \\ Dynio Honrubia, ${ }^{4,5}$ Osamu Ohara, ${ }^{7,8}$ Joe C. Adams, ${ }^{4,6}$ and Cynthia C. Morton ${ }^{1,2,4}$ \\ ${ }^{1}$ Department of Obstetrics, Gynecology and Reproductive Biology, Brigham and Women's Hospital, Boston, MA 02115, USA \\ ${ }^{2}$ Department of Pathology, Brigham and Women's Hospital, Boston, MA 02115, USA \\ ${ }^{3}$ Speech and Hearing Bioscience and Technology Program, Harvard-MIT Division of Health Sciences and Technology, \\ Cambridge, MA 02139, USA \\ ${ }^{4}$ Harvard Medical School, Boston, MA 02115, USA \\ ${ }^{5}$ Department of Neonatal Care, Children's Hospital, Boston, MA 02115, USA \\ ${ }^{6}$ Massachusetts Eye and Ear Infirmary, Boston, MA 02114, USA \\ ${ }^{7}$ Kazusa DNA Research Institute, Chiba 292-0812, Japan \\ ${ }^{8}$ Laboratory of Immunogenomics, RIKEN Research Center for Allergy and Immunology, 1-7-22 Suehiro-cho, Tsurumi-ku, \\ Yokohama, Kanagawa 230-0045, Japan
}

Received: 30 September 2003; Accepted: 15 December 2003; Online publication: 12 May 2004

\begin{abstract}
We have cloned a novel human gene, designated PFET1 (predominantly fetal expressed T1 domain) (HUGO-approved symbol KCTD12 or C13orf2), by subtractive hybridization and differential screening of human fetal cochlear cDNA clones. Also, we have identified the mouse homolog, designated Pfet1. PFET1/Pfet1 encode a single transcript of approximately $6 \mathrm{~kb}$ in human, and three transcripts of approximately $4,4.5$, and $6 \mathrm{~kb}$ in mouse with a $70 \%$ GCrich open reading frame (ORF) consisting of $978 \mathrm{bp}$ in human and $984 \mathrm{bp}$ in mouse. Both genes have unusually long 3' untranslated (3' UTR) regions (4996 bp in human PFET1, 3700 bp in mouse Pfet1) containing 12 and 5 putative polyadenylation consensus sequences, respectively. Pfetin, the protein encoded by PFET1/Pfet1, is predicted to have 325 amino acids in human and 327 amino acids in mouse and to contain a
\end{abstract}

\footnotetext{
Nucleotide sequences have been deposited in the GenBank database under accession numbers AF359381 for human and AY267461 for mouse.

(Barbara L. Resendes and Sharon F. Kuo) Co-first authors.

Correspondence to: Cynthia C. Morton - Brigham and Women's Hospital - 77 Avenue Louise Pasteur - Boston, MA 02115 - Telephone: (617) 525-4532; Fax: (617) 525-4533; email: cmorton@ partners.org
}

voltage-gated potassium $\left(\mathrm{K}^{+}\right)$channel tetramerization (T1) domain. Otherwise, to date these genes have no significant homology to any known gene. PFET1 maps to the long arm of human chromosome 13, in band q21 as shown by FISH analysis and STS mapping. Pfet 1 maps to mouse chromosome 14 near the markers D14Mit8, D14Mit93, and D14Mit145.1. The human $6 \mathrm{~kb}$ transcript is present in a variety of fetal organs, with highest expression levels in the cochlea and brain and, in stark contrast, is detected only at extremely low levels in adult organs, such as brain and lung. Immunohistochemistry with a polyclonal antibody raised against a synthetic peptide to PFET1 sequence (pfetin) reveals immunostaining in a variety of cell types in human, monkey, mouse, and guinea pig cochleas and the vestibular system, including type I vestibular hair cells.

Keywords: novel gene, intronless, GC-rich, cochlea, predominant fetal expression, tetramerization domain, unusually long $3^{\prime}$ UTR, hair cells

\section{INTRODUCTION}

The prevalence of severe to profound bilateral congenital hearing loss is estimated at 1 in 1000 births 
(Gorlin et al. 1995). About 50\% of congenital deafness is thought to be due to environmental factors, such as acoustic trauma, ototoxicity (e.g., aminoglycoside antibiotics), and viral or bacterial infections (e.g., rubella, bacterial meningitis). The remaining $50 \%$ are attributed to genetic causes and are categorized as syndromic or nonsyndromic hearing loss. Approximately $77 \%$ of hereditary deafness is estimated to show autosomal recessive inheritance, $22 \%$ is autosomal dominant, $1 \%$ is X-linked, and less than $1 \%$ segregates through the maternal lineage via mitochondria mutations (Morton 1991). Hundreds of syndromes are recognized in which hearing loss is among the clinical findings (Gorlin et al. 1995); over 90 loci have been mapped for nonsyndromic hearing loss (51 autosomal dominant, 39 autosomal recessive, 1 modifier, and 6 X-linked), and to date (Van Camp and Smith 2003) mutations in at least 53 genes that cause deafness have been identified (Resendes et al. 2002).

We undertook an organ-specific cDNA library approach to identify genes important for hearing, a method that has been used successfully to identify various genes including auditory genes (Hedrick et al. 1984; Jones and Reed 1989; Gurish et al. 1992; Cohen-Salmon et al. 1997; Soto-Prior et al. 1997; Heller et al. 1998; Jacob et al. 1998; Robertson et al. 1998). To this end, we made a human fetal cochlear cDNA library (Robertson et al. 1994) and have used two complementary methods to identify genes within the cochlear library. The first strategy, sequencing of the cDNA library, resulted in over 14,000 ESTs and revealed the presence of more than 1200 known genes, more than 2200 EST clusters also expressed in other libraries, and 700 EST clusters unique to the cochlear library (Skvorak et al. 1999; Resendes et al. 2002). Analysis of the cochlear ESTs revealed 788 genetic loci, some of which fall within intervals of mapped deafness loci and represent positional candidates for deafness disorders (http://hearing. bwh.harvard.edu). This comparative sequence analysis led to the identification of the novel gene OTOR (Robertson et al. 2000). The alternative strategy combined the approaches of subtractive hybridization and differential screening of the cochlear library and led to identification of genes preferentially expressed in the cochlea (Robertson et al. 1994). As a result of the latter strategy, several auditory genes, namely $A T Q 1$ and $C O C H$, of which the latter is novel, have been identified from the cochlear cDNA library (Skvorak et al. 1997; Robertson et al. 2000). COCH was further shown to be responsible for a sensorineural deafness and vestibular disorder, DFNA9 (Robertson et al. 1998).

Herein we present characterization of a novel human gene, PFET1, identified from the human fetal cochlear cDNA library by subtractive hybridization and differential screening, and the characterization of its mouse homolog, Pfet1. We describe expression analyses, chromosomal mapping, and immunohistochemical analyses of the human and mouse genes.

\section{MATERIALS AND METHODS}

\section{Differential screening of a subtracted cochlear cDNA library}

Human PFET1 was initially identified from a human fetal cochlear cDNA library by subtractive hybridization and differential screening techniques utilized to identify genes important for hearing (Robertson et al. 1994). The original partial cochlear cDNA was designated 2E9. Briefly, a human fetal cochlear cDNA library was subtracted with human fetal brain mRNAs by an avidin-biotin-based procedure to enrich for cochlear-expressed transcripts. Poly (A)+ RNAs from second-trimester cochlea and brain cortex were isolated and reverse transcribed to generate ${ }^{32} \mathrm{P}$-labeled cDNA probes used for differential screening of the subtracted clones to identify those clones expressed at higher levels in the cochlea.

\section{Isolation of cDNA clones}

The human PFET1 partial cDNA, which represents the 3'-most $848 \mathrm{bp}$ of the full-length cDNA, was identified initially from the human fetal cochlear cDNA library. The full-length human PFET1 cDNA was obtained in two phases. During the first phase, $4.4 \mathrm{~kb}$ of the cDNA was obtained by using the insert from the original cochlear cDNA clone as a probe to screen $10^{6}$ recombinant phage from a human fetal brain cDNA library cloned into Lambda ZAP II (Stratagene, La Jolla, CA). Filters were prehybridized and then hybridized at $42^{\circ} \mathrm{C}$ with a ${ }^{32} \mathrm{P}$-labeled random-primed (Feinberg and Vogelstein 1984) probe in $10 \%$ dextran sulfate, $4 \times \mathrm{SSC}, 7 \mathrm{mM}$ Tris- $\mathrm{HCl}$ (pH 7.6), 0.8× Denhardt's solution, and $20 \mu \mathrm{g} / \mathrm{ml}$ sonicated and denatured herring sperm DNA in $40 \%$ formamide and $0.5 \%$ SDS. Filters were washed in $0.1 \times$ SSC in $0.1 \%$ SDS at $50^{\circ} \mathrm{C}$ prior to autoradiography using XAR-5 film (Eastman Kodak Co., Rochester, $\mathrm{NY}$ ) and intensifying screens at $-80^{\circ} \mathrm{C}$. During the second phase, the remaining $1.7 \mathrm{~kb}$ of the $5^{\prime}$ end was cloned through a computer search of the accumulated terminal sequence data of human long cDNA libraries of the Kazusa DNA Research Institute (http://www.kazusa.or.jp/huge) (Ohara et al. 1997). The longest clone, which was $6.2 \mathrm{~kb}$ in size, was isolated from an adult hippocampus library and is denoted as pg00707.

The mouse Pfet1 sequence was isolated by using the open reading frame (ORF) from the human 
PFET1 to search the GenBank EST database (http:// www.ncbi.nlm.nih.gov/BLAST/, EST database). One EST (GenBank accession No. AW230625) was identified with $95 \%$ identity at the nucleotide level and contained $160 \mathrm{bp}$ of the $3^{\prime}$ end of the ORF and $300 \mathrm{bp}$ of the beginning of the $3^{\prime}$ UTR. The AW230625 EST was derived from the $5^{\prime}$ end of a mouse IMAGE clone (accession No. IMAGE: 2647463) that was obtained from Research Genetics (now Invitrogen Life Technologies, Carlsbad, CA). Together with an overlapping mouse clone (accession No. IMAGE: 5012249), the complete sequence of $3^{\prime}$ UTR of mouse Pfet 1 was determined. The remainder of the $70 \%$ GC-rich ORF was cloned from total adult mouse brain RNA using $5^{\prime}$ rapid amplification of cDNA ends (RACE; Invitrogen Life Technologies). Because the ORF is $70 \%$ GC-rich, reverse transcription was performed at $50^{\circ} \mathrm{C}$ in the presence of $\mathrm{PCR}_{\mathrm{x}}$ Enhancer Solution (Invitrogen Life Technologies). For amplification of cDNA, the following PCR protocol was performed in the presence of $\mathrm{PCR}_{\mathrm{x}}$ Enhancer Solution: initial denaturation at $97^{\circ} \mathrm{C}$ for $3 \mathrm{~min} ; 35$ cycles of $96^{\circ} \mathrm{C}$ for $30 \mathrm{~s}, 62^{\circ} \mathrm{C}$ for $30 \mathrm{~s}$, and $72^{\circ} \mathrm{C}$ for $2 \mathrm{~min}$; and final extension at $72^{\circ} \mathrm{C}$ for $7 \mathrm{~min}$. PCR fragments were TA-cloned (Invitrogen Life Technologies) and sequenced.

\section{Genomic clone}

BLAST analysis of the PFET1 nucleotide sequence identified a $109 \mathrm{~kb}$ genomic clone (GenBank accession No. AC000403) corresponding to RPCI-1 PAC clone $264 \mathrm{~J} 2$, and this PAC was obtained from Research Genetics. PAC 264 J2 contains the entire PFET1 gene.

\section{Sequence analysis}

Nucleotide sequence of partial cDNA clones was determined using an ABI PRISM dye-terminator cyclesequencing system (PE Applied Biosystems, Foster City, CA). Sequence analysis was performed using the University of Wisconsin Genetics Computer Group software (Devereux et al. 1984) and the Open Reading Frame (ORF) Finder program at the National Center for Biotechnology Information (NCBI; http://www.ncbi.nlm.nih.gov/). The cDNA insert of pg00707 was sequenced using the shotgun strategy according to procedures previously described (Ohara et al. 1997). For DNA sequencing, dye-primer or dyeterminator cycle sequencing reactions were performed using ABI PRISM cycle sequencing kits (PE Applied Biosystems) and the products were analyzed with ABI 373 or 377 DNA sequencers.

\section{Northern blot analysis}

Total cellular RNAs were extracted (Chirgwin et al. 1979) from second-trimester human fetal organs, adult surgical specimens, and adult mouse tissues. All human organs and specimens were obtained in accordance with guidelines established by the Human Research Committee at Brigham and Women's Hospital. Ten micrograms of each sample of RNA were electrophoresed in denaturing $1 \%$ agarose-formaldehyde gels and capillary-transferred overnight in 10× SSC to GeneScreen Plus membranes (NEN Life Science Products, Inc., Boston, MA) (Thomas 1980). Mouse aging brain and mouse embryonic Northern panels were obtained from Seegene, Inc. (Seoul, Korea); each lane contained $20 \mu \mathrm{g}$ of total RNA isolated from either ICR strain whole mouse embryos at different stages or whole brain at different ages.

Filters were prehybridized for $2 \mathrm{~h}$ and hybridized overnight at $42^{\circ} \mathrm{C}$ as described above with either ${ }^{32} \mathrm{P}$ labeled random-primed probe or PCR-generated ${ }^{32} \mathrm{P}$ labeled probe. Filters were washed in $0.1 \times \mathrm{SSC}$ in $0.1 \%$ SDS at $42-55^{\circ} \mathrm{C}$ prior to autoradiography using XAR-5 film with intensifying screens at $-80^{\circ} \mathrm{C}$. A human $3^{\prime}$ UTR probe was prepared via random labeling from the original 2E9 cochlear clone; a human $3^{\prime}$ UTR internal region probe was amplified using the following primers and conditions: upper $\left(5^{\prime}\right.$ TGCAAACATGCCAAGTATTTT $\left.3^{\prime}\right)$ and lower $\left(5^{\prime}\right.$ AGGCAACCAGGTCTCCTTCT 3'); initial denaturation at $97^{\circ} \mathrm{C}$ for $3 \mathrm{~min} ; 35$ cycles of $96^{\circ} \mathrm{C}$ for $30 \mathrm{~s}, 60^{\circ} \mathrm{C}$ for $30 \mathrm{~s}$, and $72^{\circ} \mathrm{C}$ for $30 \mathrm{~s}$; and final extension at $72^{\circ} \mathrm{C}$ for $7 \mathrm{~min}$. To generate radiolabeled PCR fragments representing the beginning (507 bp) and end (462 bp) of the human ORF, the following primers and the same conditions as above were used: upper (5' CCTCTCTGTCATGGCTCTGG $3^{\prime}$ ) and lower (5' TGTTCGGGCTCCGAGTAG $\left.3^{\prime}\right)$, and upper $\left(5^{\prime}\right.$ TCCTCTTCCGCTACATCCTG $\left.3^{\prime}\right)$ and lower $\left(5^{\prime}\right.$ TTGAGGTAATAGCGCGAGGT $3^{\prime}$ ), respectively.

For generation of a mouse Pfet1 ORF 460 bp probe (contains $160 \mathrm{bp}$ of the $3^{\prime}$ region of the Pfet 1 ORF and $300 \mathrm{bp}$ of the beginning of the $3^{\prime}$ UTR) from mouse clone AW230625, the following primers and PCR conditions were used: upper (5' CAGGCCTTCG ATAAGCTGTC $3^{\prime}$ ) and lower (5' CGACATCCTGA CTCTTGCAT $\left.3^{\prime}\right)$; initial denaturation at $97^{\circ} \mathrm{C}$ for $3 \mathrm{~min}, 35$ cycles of $96^{\circ} \mathrm{C}$ for $30 \mathrm{~s}, 58^{\circ} \mathrm{C}$ for $30 \mathrm{~s}$, and $72^{\circ} \mathrm{C}$ for $30 \mathrm{~s}$; and final extension at $72^{\circ} \mathrm{C}$ for $7 \mathrm{~min}$. For generation of the mouse Pfet $13^{\prime}$ UTR probe 1 (400 bp), the following primers and PCR conditions were used: upper (5' GGCTCATAGGACAGCACCTC $\left.3^{\prime}\right)$ and lower (5' GCATGGCTGCACATCAGATA 3'); initial denaturation at $97^{\circ} \mathrm{C}$ for $3 \mathrm{~min} 35$ cycles of $96^{\circ} \mathrm{C}$ for $30 \mathrm{~s}, 60^{\circ} \mathrm{C}$ for $30 \mathrm{~s}$, and $72^{\circ} \mathrm{C}$ for $30 \mathrm{~s}$; and final extension at $72^{\circ} \mathrm{C}$ for $7 \mathrm{~min}$. For generation of 
the mouse Pfet1 3' UTR probe 2 (394 bp), the following primers and PCR conditions were used: upper (5' GAGGGAATCGTTTTGATGTGA 3') and lower (5' CCCAGCAATTTATGGAGTTGA $3^{\prime}$ ); initial denaturation at $97^{\circ} \mathrm{C}$ for $2 \mathrm{~min}, 35$ cycles of $96^{\circ} \mathrm{C}$ for $30 \mathrm{~s}$, $60^{\circ} \mathrm{C}$ for $30 \mathrm{~s}$, and $72^{\circ} \mathrm{C}$ for $30 \mathrm{~s}$; and final extension at $72^{\circ} \mathrm{C}$ for $7 \mathrm{~min}$.

\section{Gene mapping}

A human PAC (246 J2, GenBank accession No. AC000403) containing the entire PFET1 gene was obtained from Research Genetics and used to generate a biotin-labeled probe for fluorescence in situ hybridization (FISH). About $3 \mu \mathrm{g}$ of PAC DNA were labeled with dNTPs conjugated with biotin (Boehringer Mannheim, Indianapolis, IN) according to the manufacturer's protocol, precipitated with $6 \mu \mathrm{g}$ of Cot-1 DNA (Gibco-BRL, Rockville, MD), and resuspended in $30 \mu \mathrm{l}$ hybridization buffer $(50 \%$ formamide, $2 \times$ SSC). Hybridization of metaphase chromosomes from peripheral blood lymphocytes obtained from a normal male was performed using $0.5-1 \mu \mathrm{g}$ of labeled probe. The biotin-labeled probe was detected using Cy3 avidin (Amersham, Little Chalfont, Buckinghamshire, UK) and chromosomes were counterstained with 4,6-diamidino-2-phenylindole dihydrochloride (DAPI) (Vysis, Downers Grove, IL). The map position of the PFET1 gene was determined by visual inspection of the signal on the DAPI counterstained metaphase chromosomes. Chromosomes and signals were observed with an Olympus AX70 photomicroscope and photographs were captured using a Photonics CCD camera and Genus software (Applied Imaging, Santa Clara, CA). Mapping of the mouse Pfet1 gene was performed by using the $3 \mathrm{~kb}$ mouse sequence to search for identical sequences in the Celera mouse genome database (Celera, Rockville, MD).

\section{Tissue preparation for immunohistochemistry}

Cochleas from three mice (6-8 weeks old), two guinea pigs (less than 3 months old), one monkey (unknown age), one adult human (68 years old), and one fetal human (20 weeks old) were used in this study. Human fetal tissues were fixed in $4 \%$ paraformaldehyde in PBS at $4^{\circ} \mathrm{C}$ for 2-3 weeks and then decalcified in $0.1 \mathrm{M}$ EDTA in PBS at $4^{\circ} \mathrm{C}$ for approximately 2 weeks. The human adult temporal bone was retrieved during autopsy; postmortem time is unknown. All tissues were prepared for paraffin sections in the following manner: animals were anesthetized via intraperitoneal injection of urethane $(1.5 \mathrm{~g} / \mathrm{kg})$, and exsanguinated through transcardial perfusion of saline with $0.01 \%$ sodium nitrite, followed by fixative. Fixatives used were formalin acetic acid (FA: $10 \%$ formalin and 1\% acetic acid in PBS) and formalin glutaraldehyde (FG: $10 \%$ formalin and $0.1 \%$ glutaraldehyde in PBS). The bulla cavity of each animal was quickly exposed and $0.2-0.5 \mathrm{ml}$ of fixative was injected slowly into the scala tympani through the perforated round window. Specimens were kept overnight at $4^{\circ} \mathrm{C}$ in their respective fixative followed by one week in $120 \mathrm{mM}$ EDTA pH 7 for decalcification. For human specimens, decalcification was performed up to a month. The decalcified specimens were dehydrated in a series of ethanol solutions and xylene baths before embedding in paraffin (Imamura and Adams 1996). Serial $8-\mu \mathrm{m}$ sections were cut and mounted on glass slides.

Human fetal tissues were obtained following guidelines established by the Human Research Committees at Brigham and Women's Hospital and the Massachusetts Eye and Ear Infirmary. The care and use of animals were in accordance with NIH's "Principles of Laboratory Animal Care" and were approved by the institutional committee on animal care at both institutions.

\section{Immunohistochemical staining}

Polyclonal antibody was raised in rabbits against a synthetic peptide corresponding to amino acid residues 256-280 of PFET1 human sequence, coupled to KLH (keyhole limpet hemocyanin) (Research Genetics). This region of PFET1 is highly conserved in mouse. Antisera were affinity purified using the pfetin peptide. In order to obtain pfetin as a positive control for Western analysis, the ORF of PFET1 was cloned into vector pET28a to express the protein (Novagen, Madison, WI). Pfetin was also extracted from various adult mouse organ tissues that were shown to contain PFET1 mRNA through Northern analysis. For a negative control, the pET28a vector only and the pET28a vector with another unrelated ORF were used. All protein was expressed in bacterial cell line BL21 (DES) (Stratagene). Immunostaining of paraffin sections was performed with the biotinylated tyramine (BT) enhancement method (Adams 1992). Paraffin sections containing the cochlear regions were deparaffinzed, hydrated, and rinsed in deionized water and PBS. Sections were blocked with 5\% normal horse serum (NHS) in PBS for $30 \mathrm{~min}$ and then incubated overnight with primary anti-PFET1 antibody diluted between $1: 1000$ and $1: 4000$ in 1\% NHS-PBS at room temperature in a humid chamber. Sections were rinsed with PBS and incubated for $1 \mathrm{~h}$ in a 1:1000 dilution of biotinylated goat anti-rabbit IgG (Vector Laboratories, Inc., Burlingame, CA) in $1 \%$ 
NHS-PBS. Sections were rinsed in PBS and incubated with Vectastain ABC reagent (Vector Laboratories). After an hour, sections were rinsed in PBS and incubated with BT diluted 1:100 in $0.01 \% \mathrm{H}_{2} \mathrm{O}_{2}$ for $10 \mathrm{~min}$, rinsed in PBS, and incubated with $\mathrm{ABC}$ reagent for another $30 \mathrm{~min}$. The primary antibody was visualized using $0.05 \%$ DAB $\left(3,3^{\prime}\right.$ diaminobenzidine) in $0.01 \% \mathrm{H}_{2} \mathrm{O}_{2}$ and $0.1 \mathrm{M}$ phosphate buffer. Controls for the immunostaining procedures were done with serial dilution of the affinity-purified primary antibody and with $1 \%$ NHS-PBS containing no primary antibody.

\section{RESULTS}

\section{Identification of human PFET1 by subtractive hybridization and differential screening}

To identify genes preferentially expressed in the cochlea, subtractive hybridization and differential screening techniques were performed using a human fetal cochlear cDNA library. Subtracted cochlear clones were differentially screened with ${ }^{32} \mathrm{P}$-labeled total fetal cochlear and total fetal brain cDNA probes, identifying those clones that were highly or preferentially expressed in the cochlea (Robertson et al. 1994). Slot blot analysis revealed that PFET1 had an increased level of expression in cochlea as compared to brain (data not shown), and led to its selection for further analysis.

\section{Nucleotide and amino acid sequence analysis}

To identify the full-length sequence of PFET1, human fetal brain and adult hippocampus libraries were screened and yielded several overlapping cDNA clones. The longest clone $(6.2 \mathrm{~kb})$ was isolated from the adult hippocampus library and represented the full-length sequence of PFET1, containing a predicted full-length ORF of 325 amino acids encoded by one exon and a 3' UTR of 4996 bases containing 12 polyadenylation consensus sequences (Figs. 1 and 2). The detailed information of the isolated clone is available on a Gene/Protein characteristic table for KIAA1778 of the HUGE database (URL, http://www.kazusa.or.jp/huge). Analysis of the upstream sequence revealed a Kozak sequence (GCCCGG CCACCN 8 ATGG) (Fig. 1). The 240 bases $5^{\prime}$ to the ORF and the entire ORF are very GC-rich (78\% and 70\%, respectively) (Figs. 1 and 2). BLAST analysis (dbNR) revealed homology to no known genes in GenBank, identified one partial human fetal brain mRNA clone designated 24475 (GenBank accession number AF052169), and one chromosome 13 PAC clone designated $246 \mathrm{~J} 2$ (GenBank accession number AC000403). BLAST analysis (dbEST) revealed homology to 279 ESTs from various human libraries, including fetal cochlea $(n=31)$, adult and fetal brain $(n=29)$, adult heart $(n=14)$, adult kidney $(n=16)$, adult lung $(n=17)$, adult ovary $(n=17)$, and adult muscle $(n=5)$. The findings of a consensus Kozak sequence, and that longer transcripts containing additional $5^{\prime}$ sequence were not revealed by RT-PCR (data not shown), library screening or, computer searching of public databases, suggest that the $6.2 \mathrm{~kb}$ clone contains the entire gene.

One mouse EST (AW230625) with 95\% identity at the nucleotide level and containing part of the ORF (170 nucleotides of the $3^{\prime}$ end of the ORF) was identified from GenBank (mouse dbEST). The remainder of the mouse Pfet1 ORF was cloned from mouse brain total RNA by performing $5^{\prime}$ RACE. The remaining $3.4 \mathrm{~kb}$ of the mouse Pfet $13^{\prime}$ UTR was obtained by sequencing IMAGE clone 5012249 that overlapped with mouse AW230625 EST (see Materials and Methods). Like its human homolog, the mouse Pfet1 gene has an unusually long 3' UTR (3700 bp) and contains five putative polyadenylation consensus sequences (Fig. 3).

The protein domain database, Pfam (Bateman et al. 2000), was used to identify protein motifs or domains in the predicted amino acid sequence. A voltage-gated potassium channel tetramerization (T1) domain of 95 amino acids, spanning amino acids $34-129$, was identified $(E=7.3 e-17)$ in the amino terminal region (Figs. 1 and 4 ). No other complete domains were predicted. A hydropathic profile utilizing the Kyte-Doolittle method revealed four weakly hydrophobic regions of at least 10-15 amino acids (data not shown). The deduced amino acid sequence does not appear to contain any transmembrane spanning domains, as determined by the transmembrane prediction programs TMpred (Hofmann and Stoffel 1993), TMHMM (Sonnhammer et al. 1998), and HMMTOP (Tusnady and Simon 1998).

The human PFET1 and mouse Pfet 1 genes are 91\% identical at the nucleotide level within the ORF and share little sequence similarity outside of the ORF (Fig. 2). The mouse ORF is longer by two amino acids (insertion of proline and histidine at positions 142 and 143 , respectively) and the predicted protein differs by eight amino acids (Fig. 2). The human and mouse pfetin are predicted to have 325 amino acids in human and 327 amino acids in mouse and to contain a voltage-gated potassium channel tetramerization domain. The entire human and mouse ORFs are very GC-rich $(70 \%)$. The tetramerization domain in the mouse Pfet 1 is identical to that of the human PFET1 except for a phenylalanine-to-leucine change at position 88 (Fig. 2). 
GCGGGCAAGGCGGGCGGAGCGCACTGGAACTCAAGGGGGCGCACAGCGGC GCGCTCGCACCGCTCGGCTCCGCGCGGCTCTAGGAGGTGGCGGCGGTGGC GGTGGCGGCGGTGGCGGCGGCGGCGGCGGCGGGGCGCAGGGCTGAGCGAG CGTCCGGGTTCCGGGGCTCCGGGGAAGGCGGTTGCAGCTCCTGAGTGCAG CGCGGCTTCCTGCCACTGTCCCGGCCCGGCCACCTCTCTGTC

1 atggetctggcggacagcacacgtggattacccaacgggggcggc

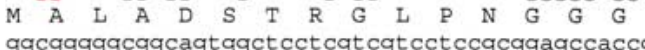

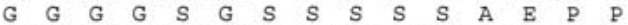

90 ctcttccceacatcgtggagetgaacgtggggggccaggtgtac $\begin{array}{lllllllllllllll}\text { L } & F & P & D & I & V & E & \text { L } & N & V & G & G & Q & V & \text { Y } \\ \text { gtgacccoggegctgcacggtggtgtcggtgccogact cgctgctc }\end{array}$ \begin{tabular}{lllllllllllllll}
$V$ & $T$ & $R$ & $R$ & $C$ & $T$ & $V$ & $V$ & $S$ & $V$ & $P$ & $D$ & $S$ & $L$ & $L$ \\
\hline
\end{tabular}

180 tggcgcatgttcacgcagcagcagccgcaggagctggccegggac \begin{tabular}{lcccccccccccccc}
$W$ & $R$ & $M$ & $F$ & $T$ & $Q$ & $Q$ & $Q$ & $P$ & $Q$ & $B$ & $L$ & $A$ & $R$ & $D$ \\
\hline agcaaaggccgct tctttctggaccgggacggettcctcttccgc
\end{tabular} \begin{tabular}{llllllllllllllll}
$S$ & $K$ & $G$ & $R$ & $F$ & $F$ & $L$ & $D$ & $R$ & $D$ & $G$ & $F$ & $L$ & $F$ & $R$ \\
\hline
\end{tabular}

270 tacatcctggattacctgcgggact tgcagctcgtgctgcecgac \begin{tabular}{llllllllllllllll}
$Y$ & $I$ & $L$ & $D$ & $Y$ & $L$ & $R$ & $D$ & $L$ & $Q$ & $L$ & $V$ & $L$ & $P$ & $D$ \\
\hline tact tecccgagcgcagceggetgcagcgegaggccgagtacttc
\end{tabular} $\begin{array}{llllllllllllllll}Y & F & P & E & R & S & R & L & Q & R & E & A & E & Y & F & (120)\end{array}$

360 gagctgccagagctcgtgcgccgectcggggegcccagcagccc $\begin{array}{lllllllllllllllll}E & L & P & E & L & V & R & R & L & G & A & P & Q & Q & P & \text { (135) }\end{array}$ 405 ggeccggggecgecgecetcgcggegcggggtgcacaaggagggc $\begin{array}{llllllllllllllllllll}G & P & G & P & P & P & S & R & R & G & V & H & K & E & G & \text { (150) }\end{array}$

450 tcgetgggtgacgagetgetgecget tggetactcggagcccgaa S L L G

495 cagcaggagggegectctgecggggcgecgtcgeccacgetggag

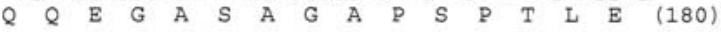
540 ctggetagccgcagtccgtccgggggegcggegggeccgetgetc $\begin{array}{lllllllllllllllll}\text { L } & \text { A } & \text { S } & \text { R } & \text { S } & \text { P } & \text { S } & G & \text { G } & \text { A } & \text { A } & \text { G } & \text { P } & \text { L } & \text { L } & \text { (195) }\end{array}$

585 acgecgtcccagtcgetggacggeagccggcgetcgggetacatc $\begin{array}{lllllllllllllll}T & P & S & Q & S & L & D & G & S & R & R & S & G & Y & I \\ \text { accatcggctaccgcggetcctacaccatcgggcgggacgcgcag }\end{array}$ T I G Y R G 675 gcggacgccaagttccggcgagtggcgcgcatcaccgtttgcgga $\begin{array}{lllllllllllllllll}\text { A } & D & A & K & F & R & R & V & A & R & I & T & V & C & G & (240)\end{array}$

720 aagacgtcgetggecaaggaggtgtttggggacacctgaacgaa $\begin{array}{llllllllllllllll}K & T & S & L & A & K & E & V & F & G & D & T & L & N & E & \text { (255) }\end{array}$

765 agcegggaccecgaccgtcceccggagegctacacctcgegetat

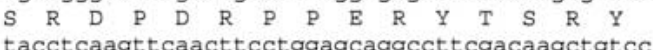
$\begin{array}{lllllllllllllllll}Y & \text { L } & K & \text { F } & \text { N } & \text { F } & \text { L } & \text { E } & \text { Q } & \text { A } & \text { F } & \text { D } & \text { K } & \text { L } & \text { S } & \text { (285) }\end{array}$ 855 gagtcgggcttccacatggtggegtgcagctccacgggcacctgc $\begin{array}{lllllllllllllllll} & \text { E } & S & G & F & H & M & V & A & C & S & S & T & G & T & C & (300)\end{array}$

900 gectttgccagcagcaccgaccagagcgaggacaagatctggacc

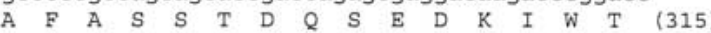

945 agctacaccgagtacgtcttctgcagggagtga 1220

$$
S \text { Y } T \text { T E Y V F C R E * (325) }
$$

978 GCTCCCCAGACCCCCTCGCCACTCCAGCGCCCAGTCCTTCTCCTGCCCGA 1028 GAGATGATTACAGAGCCTCTTGTCCCACCTTTGTCCCCTGGCTGCTGCCC 1078 TCCCATTCTCCCCCTCCAGTAGTAGCTGGGTGAGACCTGTCCGCCCACCT 1128 TCCCTCCACTACAGAACCTGCAGCCGCAAATCCTCTGGGCTGCTTCGTCT 1178 TCTTTGGACCTCCTGAACCGAGAGAACCCAGAGGAACCCCCACCCCACCC 1228 CCACCTACCACTCCATGCTTTCTCTACTCCCTGCCTCAAACCACCCCTCC 1278 CCCAGATGGTACTTCAGTTTGGATCTATTGGGGGAGTGTGGCCACAGACC 1328 GGGGGATGATTGAATTGTTCAGAACCTGATTGGACCGTGTCCAATGTGCG 1378 GAAGATTTCCTTGAAATCTTCTCAAGCTCTTATGACTCACTGGGGGTTTA 1428 AGAGATCAGGATTGGTTCCACTGTCTGGGGTTAGTGTTTTACAAGGTCAT 1478 TACACAGTCTTTTTGACCTCTTTTGAAGGTAGAGTTTTAGAAGGCTGGAT 1528 GGAAGATTCTGAGCCTGGAATTAGGACCCCATGGAGGCAGTTCAGTAACT 1578 AAACTAATAAAGTTTTGAAAAGTTACACGTAAAGTAGAAGAATCTAGTGC 1628 GTGGGACAGTAAAGGATCCTTTCTCGTACAGAATAAAAGGTCTCAGCCTG 1678 TAGCTTAAACTTATAGAAAGTGATCCGCCTGCCTGCAGAGGCGCCCTTTT 1728 CAGCTGCTGCTCGCCAGAAGCCCTTGATTCCACTGGTTGACATGGCAGCA 1778 GTTACTGGCAAGAGGGAGAAAGGACGCTGCCGCCTAAGAGTGCAAGGCTG 1828 CTCAGGTCTCCAAGCGCCGTAGGAGGTCACCTGGCAGTGACTGTAGGGAG 1878 CTGGGTCATAGTGCACGTCGTGGGTATTAGGAAAGCCTGTATTCTTTCAA 1928 TGAATGTCAGTAGGACCTTCCTTTAGCTGTAAGACTTGGTGGGCGGGGTG 1978 GGGTGGGGAGGGAGGAAAGGGTAGGAAGGGTGGGAAGGGAGAAGCAGACA 2028 TAGTCATTTATGATTTGAAAGTTGGAAGTTTGTACCATCTGTTTGAGTAT 2078 ATGCACATTTAAAAAAATATCATATAGTAAATGCAAACATGCCAAGTATTT 2128 TATAAAGATTAATAACAGACCTACTCTTACCTGGCAGTTTACTTAACTTA 2178 CTGTTTTGAGTCCTAAACTTAGAGTTGTTAATGCTTATATATAATCTAAC 2228 CAAAGAGTTACCCAGTAGGGTTTTAGTTTTTGAACTTTTATTTTCTTGTT 2278 GATTATAAATCCTGATTTTGGAATCTATTGCGCAAAAGAAGTTTCATTTT 2328 GGTTACTTAGACCTAAGATCACTTATTAAAAATCCTTATTTTCTCCAAGC 2378 CCAGCAAACGTTGACTTCTGGGCAAACCTGAAAACCTGAAAATGCCACTT 2428 TCATGCAGTTTGTTTGAAGTTAAGTGGAATCCTTTCAAATGACGAGCTGC

FIG. 1. Nucleotide sequence of human PFET1 cDNA and its deduced amino acid sequence. Nucleotide numbers are shown on the left and amino acid sequence numbers are shown in parentheses on the right. The Kozak consensus sequence is outlined in red. The
2478 AGAGAACTCAGCACCAAGGGCTGCCTATCTGTAGATAGCTGTAAAATGGA 2528 ATATTTTTAAATGAAGGCAAATAAGTACTTAAAAGTGAGCTGAGCAATA 2578 ÄATGGTCCAATAATAGGTAAATGCAACAGAAACAGAAGGAGACCTGGTTC 2628 CCTTATGCCTTTACTCTTACATGGAATAAATTCCCAATGCATATCCTATC 2678 TAAACCATAAGTGAAGGGAAATAAACCTCGTCATGCTCCATGCTGTGAGG 2728 TGTCCTTTGGATATTCTGTGATGACAGAGAAGCCTATTTTGTTTTGTTTT 2778 CAGCATCTTTCTCTGATGTACGTTTTTAAGGATTTTGTAAGAGCTGTTTT 2828 CAGTGTTTAAATTAGTGCTATTTTTCCTTGTTTTTAAAAAATGAATCTCGT 2878 ACTGTATCTTACTATGTCCATACAGATGTTACAAATCGACAGTTTTATTC 2928 TTAGACTCATGTGATCCAAGCTGTATATACCATATATAAACATTTTACAT 2978 GAATCATTTAGTTTTTTAATTCATTTACTAATGCTATAAAATTTCCTATA 3028 TTACCCCAGTAATTTGCATCAGCTGGTTTATATACTAAAGCAACATGTTT 3078 TGATGAGTTTCTTACATCCTTATCGAGGAATTGGGTTAGGAAAAAATACA 3128 TAATTGTAAAACTGAGTTTGCTGTATTATACTTTTTTTCTTGAGTATTAG 3178 TTGTATTACTAATCATATGTTGATTAACTGTCTACTTAAAGTCAAGGTAC 3228 CTGTATTTTTAATCCACTAATTTTTTTTTAAGTTGGGAAATAGATTTCAAG 3278 TCTTTTATTAGACTAACATTTTTTGAGAAGTAAAATTGACTTTATATACA 3328 AAGCCTGTAATTTTAGGCGAAATGGAAGCAGAAATCTAGGAAGTTGTGCT 3378 TGCTTGTATGGTCTCAGACTAAGTAATGCATCAGAATTCATCTGTTTGAA 3428 GCCTGAAATAATTTAGGACTCTGATTCACTGACCAAAAGTCAGTGTTGCA 3478 GAGATTTCTCTACCCCGTATGGTATTTTGTTAGATTGTTCAACAGGAAGC 3528 ACATGATTGAGAACATCTTGGGACAGACCAAAACCACTGACAGATGGCAA 3578 GGCTCGGCGATTCTGATTTCCCTTCTCAAATCTGCTCAACTCCAAGAGTC 3628 TTGAGAAACTGCTAAAATTTTGCCTCTGTCACTCAAGTCTTACAAATGTT 3678 ATCTTGTAAACCTTTGAGGTGAACTATTCCACTGTCTTGTACATAGGCAT 3728 CTTATTCACTGCACCCTGTCACACCCAGCACCCCCCGCCCCGCACATTAT 3778 TTGAAAGACTGGGAATTTAATGGTTAGGGACAGTAAATCTACTTCTTTTT 3828 CCAGGGACGACTGTCCCCTCTAAAGTTAAAGTCAATACAAGAAAACTGTC 3878 TATTTTTAGCCTAAAGTAAAGGCTGTGAAGAAAATTCATTTTACATTGGC 3928 TAGACAGTAAAAAAACAAGTAAAATAACTTGACATGAGCACCTTTAGATCC 3978 CTTCCCCTCCATGGGCTTTGGGCCACAGAATGAACCTTTGAGGCCTGTAA 4028 AGTGGATTGTAATTTCCTATAAGCTGTAATAGTGGAGGTATTGTGGGTTC 4078 ATTTGAGTAAGCCCTCCAAAGATACCATTCAAATAACCTGGGAGAATGTC 4128 ATAAATTATTCAGATAATTATCACTGCATGAATCTGATTCAGAGGCATGC 4178 ATTTACATATGTTGCCCTAATTACCATTTGATGATCATAAATACAAGTGA 4228 ATGACATTGGACTTTTAGTAACAAACTTAATTTTTAAAAAAGTGTAGACA 4278 ATGGTGGTTAAAAAAAAAAAAAAAAAAGGTGCCAGGTTCTGTGTGTTTGCAC 4328 CAAGTAATTGACATGTTTTTTGTTTAATACATGTGGACCATGAACAGTAT 4378 TCATTCTACTTTTTCAAATGATATGCTGTAGAAAATATTCCTTGAAGATG 4428 TGAGATTTAAAAAATTTTCCCTTTCAATGTTGTTTTAATTGTATTTCTTA 4478 CTTGGTTTTTTTGATTGATAGCACAGTGATAAATCATAATACTAGACAAA 4528 ATTGTCTTCTCTTTCAAACCAGAGCCATATATATGTCTGTATATATGGGA 4578 CCTACTGCTTCTCTGAGGAAATGCATAATCTGTTAATATCAGACAAAATG 4628 AGCAATTGGCAGTGCTCATAATATATTCCAATTTTTATTGGAATTTTCGA 4678 TGGAATGTTATTTCAATAAAGCCATGTAAGGTGAAACTTTGATAACTTTT 4728 TACTCTTCAAGTTAGGGTAAATTCTGATCCAATATTCAATTCATTTGTGT 4778 ACTCCCACATGCAAAATGCTAAATTACAATGCAGACATTAAGAAAAAGTA 4828 TTGACTGGAGGGGTTGAATTCCTTGAGAATTTATTTTATAGTCTAAATCA 4878 CAAATACTTTACTCAATTTAGTTTTTAAAATAGTAAACTGAATATTTTTG 4928 TTGTAAGCCTATCAGAGTCAATCCTTCGTTTGGAATTGTTTTCCTGTTTT 4978 TCCTTACTATAAATCATTTAAAAAACTGAATTCATTTTCTTAGATGGCATA 5028 AGTCTGTCTCTTGAGAAATAAGTAAAATACTCCTATTTTCAGTATCTGTA 5078 GCACCTGAAATAGGTCTTTGTATAGCCAGAAACAAGTTATGTTGAAGTTA

5128 GCTTTTCTTTGTCAACAGTTTTGGACAATAAAAATCTGAAAGTATTAACA 5178 CTTGATTTTCTACTGGGGCCCTTCAAACTTGGTTGGAAGAAATTCAACCA 5228 GAATATCTACATTAGAGTATAATCATGTGTGGTAGGAAGATGGACTAGTT 5278 AATCAAGATTTGTTGTCACTTAAATTTTTTGTGATTTTTTTCCAAGCCAG 5328 TTTTTTTAAATTCTAAATGTGTTTTGAGGTATGGGTACATTAATTGTAAT 5378 GTAAACTATTATACAACTGTTTTTGCGACTTTATAGGCAGGTAAATTTTG 5428 CTATTACTATTGAATACAAATGACAATTCATTTATGACCACTCAAACAGC 5478 GTTAGTAACCATTTAGTGACAAAGGATTAAAACATCCATCTGGATGTTAA 5528 TTTTGAAGATGTAAATTATATGTTGTTTAAATTTTTCCAGGCATCTGAAA 5578 ACCTTATCTGCTAGACAATGTAAGATTCACACAGAGTTATCTGGGATTCT 5628 GATTTTTTAAATAGTACATATCATTAAACCATTTTCTCTAAATGTAAGAA 5678 GAGCAGAAAAAATCTTATAAGATTATCAGATTTTTCTAATGACACAGAAA 5728 TGTAAGAAAAAAAATCCCTTTATATTGAAAAAAGATGCAGTCAAAGTCTTT 5778 TCAGACATGCCCAAACTTTGAGAATTCCTTCAACCATCTAATGCTATAAA 5828 GATTTTTGTTCTTCCTGTTCACAACCAGTTGTATAACAGAAATACTAGCT 5878 ACTGTTTTCCTTCCTGTGTGTGAAGTAATGAATCATTGATTATGTGACTT 5928 GTTATGTATTCAATTAAACACTAAAGAATAAAACATTCACTCCTTT 5978

tetramerization domain is underlined. An asterisk denotes the stop codon. Putative polyadenylation consensus sequences are shaded. The boxed sequence represents the original clone obtained from the cochlear cDNA library. 
ATGGCTCTGGCGGACAGCACACGTGGATTACCCAACGGGGGCGC

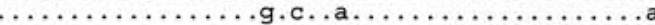

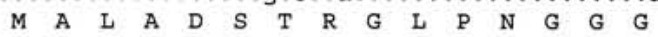

GGCGGGGGCGGCAGTGGCTCCTCGTCGTCCTCCGCGGAGCCACCG .................................

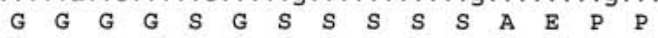

CTCTTCCCCGACATCGTGGAGCTGAACGTGGGGGGCCAGGTGTAC .................................

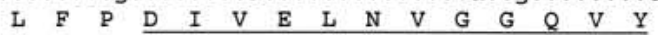
GTGACCCGGCGCTGCACGGTGGTGTCGGTGCCCGACTCGCTGCTC

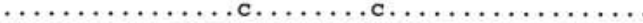

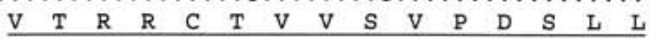
TGGCGCATGTTCACGCAGCAGCAGCCGCAGGAGCTGGCCCGGGA

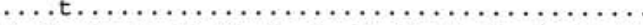
\begin{tabular}{llllllllllllllll} 
W & $R$ & $M$ & $F$ & $T$ & $Q$ & $Q$ & $Q$ & $P$ & $Q$ & $E$ & $L$ & $A$ & $R$ & $D$ \\
\hline
\end{tabular}

AGCAAAGGCCGCTTCTTTCTGGACCGGGACGGCTTCCTCTTCCGC

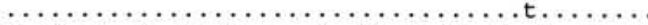
\begin{tabular}{llllllllllllllll}
$S$ & $K$ & $G$ & $R$ & $F$ & $F$ & L & D & R & D & G & F & L & F & R \\
\hline &. &. &. &. &. &. &. &. &. &. &. & F &. &.
\end{tabular}

TACATCCTGGATTACCTGCGGGACTTGCAGCTCGTGCTGCCCGAC

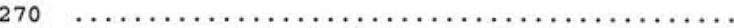
(91) $\begin{array}{lllllllllllllllll} & \text { I } & \text { I } & \text { L } & \text { D } & \text { Y } & \text { L } & \text { R } & \text { D } & \text { L } & \text { Q } & \text { L } & \text { V } & \text { L } & \text { P } & \text { D }\end{array}$
TACTTCCCCGAGCGCAGCCGGCTGCAGCGCGAGGCCGAGTACTTC

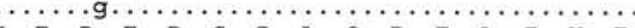
GAGCTGCCAGAGCTCGTGCGCCGCCTCGGGGCGCCCCAGCAGCCC

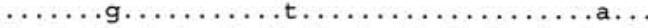
$\begin{array}{lllllllllllllllllllll}E & \text { L } & \text { P } & \text { E } & \text { L } & \text { V } & R & R & \text { L } & G & A & P & Q & Q & P\end{array}$ GGCCCGGGGCCGCCGCC - ...- TCGCGGCGCGGGGTGCACAAG

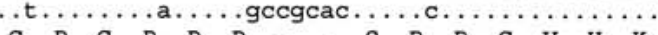

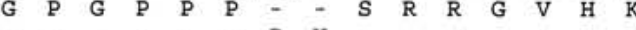
GAGGGCTCGCTGGGTGACGAGCTGCTGCCGCTTGGCTACTCGGAG

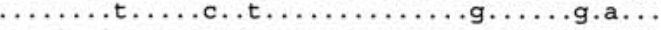

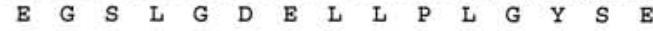
CCCGAACAGCAGGAGGGCGCCTCTGCCGGGGCGCCGTCGCCCACG

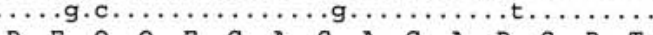
CTGGAGCTGGCTAGCCGCAGTCCGTCCGGGGGCGGGCGGGCCCG

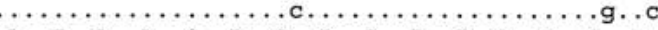

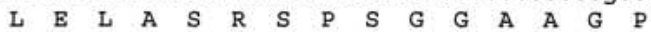
CTGCTCACGCCGTCCCAGTCGCTGGACGGCAGCCGGCGCTCGGGC

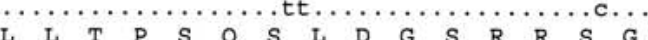
TACATCACCATCGGCTACCGCGGCTCCTACACCATCGGGGGGAC

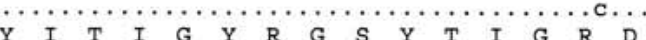
GCGCAGGCGGACGCCAAGTTCCGGCGAGTGGCGCGCATCACCGTT

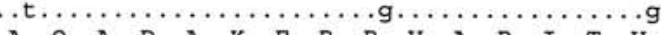

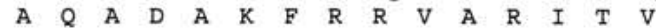

TGCGGAAAGACGTCGCTGGCCAAGGAGGTGTTTGGGGACACCCTC

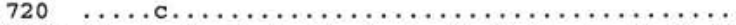
(239) C $G$ G

AACGAAAGCCGGGACCCCGACCGTCCCCCGGAGCGCTACACCTCG

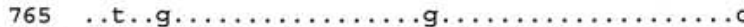
(254) $N$ E S R D $P$ D $R$ P $P$ E $R$ Y $T$ S (256)

CGCTATTACCTCAAGTTCAACTTCCTGGAGCAGGCCTTCGACAAG

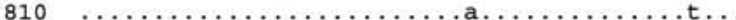

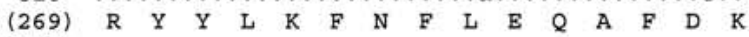
(271)

CTGTCCGAGTCGGGCTTCCACATGGTGGCGTGCAGCTCCACGGGC

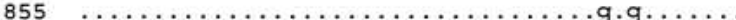
(284) L S B S G F H M V A C S S T G (286)

894 ACCTGCGCCTTTGCCAGCAGCACCGACCAGAGCGAGGACAAGATC

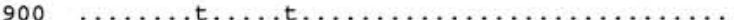
(299) T C A F A S S T D Q S E D K I (301)

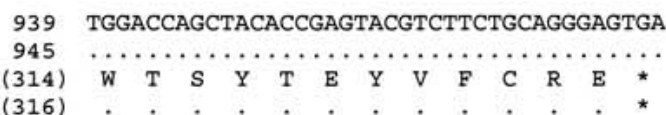

FIG. 2. Alignment of the complete deduced sequence of the open reading frames of the human and mouse Pfet 1 genes. The deduced amino acid sequences of the ORFs share 91 similarity and differ by eight amino acids, one of which is within the tetramerization domain (position 88). A high degree of sequence similarity indicates cross-species conservation of the PFET1 gene. Nucleotide sequence is capitalized for the human PFET1 gene and in lower case for the mouse Pfet 1 gene. Amino acid sequence is capitalized and numbered in parentheses. Dots represent sequence identity between human and mouse genes. Dashes represent gaps introduced to align the sequences. An asterisk denotes the stop codon. The tetramerization domain is underlined. 
ACTCGCCTGGAGCGCGCGGGCGAGGCAGGCGCAGCGCACCGGGGC TCTCGTGGGCGCACTGCTGCGCGCTCGCACCGCGCGGCTCTCAGT GGCCGGCCGCCGCCTGAGCGCAGGGCTCCCCGATAAGAGCCGCTG GGGCTTCCGATCGCGACCCCGCTCCCTGCCACTTGGCCCATCCGG GCCACCTCTTTTTGGCC

1 atggctctggeggacagcgeccgaggattacccaacgggggegga

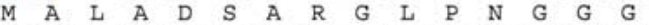

45 ggcggaggtggeagcggetcgtcgtcgtcctcggcggagccgecg G G G G S G S S S S S A E P P

90 ctcttcccggacatcgtagagctgaacgtgggagggcaggtgtat

135 gtgacccggcgctgcaccgtggtgtccgtgccogactcgctgctc \begin{tabular}{lcccccccccccccc}
$V$ & $T$ & $R$ & $R$ & $C$ & $T$ & $V$ & $V$ & $S$ & $V$ & $P$ & $D$ & $S$ & $L$ & $L$ \\
\hline tggcgtatgttcacgcagcagcagccgcaggagctggccegggac
\end{tabular}

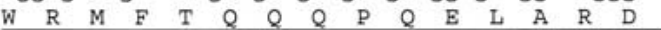

225 agcaaaggccgettctttctggaccgggacggcttcttcttccgc \begin{tabular}{lllllllllllllll}
$S$ & $K$ & $G$ & $R$ & $F$ & $F$ & $L$ & $D$ & $R$ & $D$ & $G$ & $F$ & $F$ & $F$ & $R$ \\
\hline
\end{tabular}

270 tacatcctggattacctgcgggacttgcagctcgtgctgcccgac \begin{tabular}{lllllllllllllll}
$Y$ & $I$ & L & $D$ & $Y$ & $L$ & $R$ & $D$ & $L$ & $Q$ & L & V & L & P & D \\
\hline tacttcccggagcgcagccggetgcagcgegaggecgagtacttc
\end{tabular}

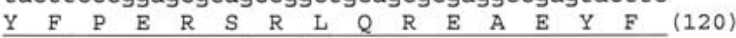

360 gagctgecggagctcgtgegtcgectcggggegecccagcaaccc

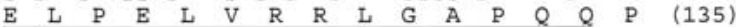
405 ggtccggggccaccgccgccgcactcgcgccgcggggtgcacaag $\begin{array}{lllllllllllllllllll}G & \text { P } & G & \text { P } & \text { P } & \text { P } & \text { P } & \text { H } & S & \text { R } & \text { R } & \text { G } & \text { V } & \text { H } & \text { K } & \text { (150) }\end{array}$

450 gagggetctctgggcgatgagctgctgccgctgggetacgcagag E G $S$ L

495 cccgagccgcaggagggcgectcggccggggegcettcgeccacg $P$ E P Q E G A S A G A P S P

540 ctggagctggctagccgcagcccgtccgggggcgcggcggggecc L E L A S R S P S G G A A G P (195)

585 ctgctcacgccgtcccagtctttggacggcagccggcgetccggc $\begin{array}{llllllllllllllll}\text { L } & \text { L } & \text { T } & \text { P } & \text { S } & Q & S & \text { L } & D & G & S & R & R & S & G & \text { (210) }\end{array}$ 630 tacatcaccatcggctaccgcggctcctacaccatcgggcgcgac $\begin{array}{lllllllllllllllll}Y & I & T & I & G & Y & R & G & S & Y & T & I & G & R & D & \text { (225) }\end{array}$

675 getcaggcggacgccaagttccggcgggtggegcgcatcaccgtg A $Q$ A $\quad$ D A $\quad$ K F $R$ R

720 tgcggcaagacgtcgctggccaaggaggtgtttggggacaccetg

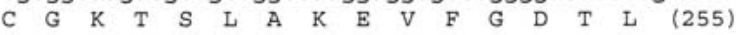

765 aatgagagecgggaccccgaccggeccceggagcgetacacctcc $\begin{array}{lllllllllllllllll}N & \text { E } & S & \text { R } & \text { D } & \text { P } & \text { D } & \text { R } & \text { P } & \text { P } & \text { E } & \text { R } & \text { Y } & \text { T } & \text { S } & \text { (270) }\end{array}$

810 cgctattacctcaagttcaacttcctagagcaggcettcgataag $\begin{array}{lllllllllllllllll}R & Y & Y & \text { L } & \text { K } & \text { F } & \text { N } & \text { F } & \text { L } & \text { E } & \text { Q } & \text { A } & \text { F } & \text { D } & \text { K } & \text { (285) }\end{array}$

855 ctgtccgagtcgggettccacatggtggcgtgcaggtgcacgggc

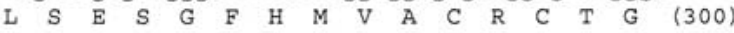

900 acctgcgcttttgctagcagcaccgaccagagcgaggacaagatc $\begin{array}{lllllllllllllll}T & C & A & F & A & S & S & T & D & Q & S & E & D & K & I\end{array}$

945 tggaccagctacaccgagtacgtcttctgcagggagtga 983

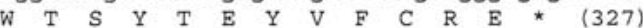

984 GCTCCCCACGCCCGCCCCTCGCCACTCCGCCGCTGGCAACAATAGCAACA 1034 GCCTGAGTGTCAATAACGGGGTTCCCGGCGGGGCCGCCGCGGCCTCCGCC 1084 ACCGCCGCCGCCGCCCAGGCCACCCCCGAGCTGGGCAGCAGCCTCAAGAA 1134 GAAGAAGCGGCTCTCGCAGTCCGATGAGGATGTCATTAGGCTCATAGGAC 1184 AGCACCTCAATGGCCTAGGGCTCAACCAGACTGTTGATCTCCTCATGCAA 1234 GAGTCAGGATGTCGTTTAGAGCATCCTTCTGCTACCAAATTCCGAAATCA 1284 TGTCATGGAAGGAGACTGGGATAAGGCAGAGAATGACCTGAATGAGCTAA 1334 AGCCTTTAGTGCATTCTCCTCACGCTATTGTGGTAAGAGGCGCACTTGAA 1384 ATCTCTCAAACGTTGTTGGGAATAATTGTGAGGATGAAGTTTCTGCTGCT 1434 GCAGCAGAAGTACCTGGAATACCTGGAGGACGGCAAGGTCCTGGAGGCAC 1484 TTCAAGTTCTACGCTGCGAACTGACGCCGTTGAAATACAACACCGAGCGC 1534 ATCCATGTCCTTAGTGGGTATCTGATGTGCAGCCATGCCGAAGACCTACG 1584 GGCAAAAAGCTGAATGGGAAGGCAAGGGCACAGCGTCCCGGTCCAAACTGC 1634 TGGACAAGCTTCAGAACTCTCCTGCGGCAGGCGGTGGAACTACAAAGGGA 1684 TCGGTGCCTATATCACAATACCAAACTTGACAATAATCTAGATTCTGTGT 1734 CTCTACTTATAGATCATGTTTGTAGTAGGAGGCAGTTTCCCTGTTACACT 1784 CAACAGATACTTACAGAGCATTGTAATGAAGTGTGGTTCTGTAAATTTTC 1834 TAATGATGGCACTAAACTAGCAACAGGATCAAAGGATACCACAGTTATCA 1884 TATGGCAAGTTGATCCGGATACGCACCTGTTAAAACTGCTTAAAACGTTA 1934 GAAGGACATGCGTATGGTGTCTCTTATATAGCATGGAGTCCAGATGACAG 1984 CTATCTTGTTGCTTGTGGTCCAGATGACTGCTCTGAGCTTTGGCTTTGGA 2034 ATGTACAGACGGGAGAATTAAGAACAAAAATGAGCCAATCTCATGAAGAC
2084 AGTTTGACCAGTGTGGCCTGGAATCCAGATGGGAAACGCTTTGTGACGGG 2134 AGGTCAGCGTGGTCAGTTCTACCAGTGTGAAAAGGACGGAAATCTTCTGG 2184 ACTCCTGGGAAGGAGTGCGAGTACAGTGCCTGTGGTGCTTGAGTGACGGG 2234 AAGACTGTGCTGGCTTCCGACACGCACCAGAGAGTCCGGGGCTACAACTT 2284 TGAGGACCTGACAGATAGAAACATAGTACAGGAAGATCATCCTATTATGT 2334 CATTTACTATTTCCAAAAATGGCCGATTAGCTTTGTTAAATGTAGCAACT 2384 CAGGGAGTTCATTTATGGGACTTGCAAGACAGAGTTTTAGTAAGGAAATA 2434 TCAAGGTGTTACCCAAGGGTTTTATACAATCCACTCGTGTTTTGGAGGCC 2484 ATAATGAAGACTTCATTGCTAGTGGCAGCGAAGATCACAAGGTTTACATC 2534 TGGCACAAACGTAGTGAACTGCCAATTGCGGAGCTCACAGGGCACACGCG 2584 CACAGTAAATTGTGTTAGCTGGAACCCACAGATTCCATCCATGATGGCCA 2634 GTGCCTCAGACGATGGCACTGTTAGAATATGGGGACCAGCACCTTTCATA 2684 GACCACCAGAATATTGAAGAGGAATGCAGTAGCATGGATAGTTGATGGCA 2734 AATTTGGAGCAGACGACTTCTGTTTAACTTAAAATTAGTCGTATTTTAAT 2784 GGCTTGGGATTTGGTGCAAACAAACATGATTGATAGCTGGACAGACATGC 2834 TCGTCATGAAAAAAAAAAAAAAAAAAGAACCATTTTGAAGCCCGATTGGGGCC 2884 AAACATTTACACCTTGCTTCCTAGTAACCAGTTGATGAAGCGCGTCTAGA 2934 ACGTTGTTGGACACCATGTTGAATTATTCCCCATCGGTTGTGAAGAACTG 2984 TGCTACATTCAGGCTTACCCATTGAACTCAGTATATATATTTTTTCCCTC 3034 CTGCCTTTTGTCTGGTGGGACACCATTCTTGTTGCTCTTCTGTGTAATGA 3084 AGTTCAATGCTTGTTTGGAAACTTTATTTAACANTTTAAAAGGCTTGATA 3134 GGAAGAGGTCATTAATCTTGAAGAGTTNCATTTGGAAGGAAAAAATTTCC 3184 TTTCGTTTCTCCAATCTTTCCCCNTTTTTANCGTGAGATCTTTGCAGCCT 3234 TGGTNCTGGATTCTAGCCTTGCCCGTTGCGCAGTATATGCNTGATCAGAT 3284 GATAAACCAGTGAACTATGTCAAAAGCACTCTCAATATTACATTTGACAA 3334 AAAGTTTTGTACTTTTCACATAGTTGTTGCCCCGTAAAAGGGTTAACAGC 3384 ACAATTTTTTAAAAATAAATTAAGAAGTATTTATAGGATTAAAGTGACTT 3434 CATTTGTATACATTTGGAATCTAAACCAGCTTAAAAACAGTGTCCTCTGT 3484 GACTGAGATATGCAGTGTAACTGATGCTCTTCTGGAGTGCCACGTGAGAC 3534 ATGGCATGGTCAGAAACAGTGTTCAGAAGGACACGGCACAGGAAAGCCAG 3584 AGAGATACTTTCCCCTTTTTATTTTATTCCTGAAGGGACATCAGTACCTG 3634 ATACTGAAGAAATTCAAGATTCAAAAGGAAAATTTTATAAATAAACCAGT 3684 ACAGAAGATCAGCATCAGTCTAGGTTTTCAAGAAAGCTTGTTCCAAGTTC 3734 TCTGAACTTGAGGGAATCGTTTTGATGTGATCTANCANAAGTAGACATCA 3784 NAAGATAGACCTACTTTGGGAATTTATAGTGTAGTTAAATCTTAGAGGAA 3834 GTCAGCAGGCTACAAACATTTATGTAAACTGGAATTAAAGCCTGAGTCAT 3884 TTCTCCTAATTGCCCTTAATGTCCAACATATAGGGACACTCATTTAAAGA 3934 AGATTCCTTCTCAGCTTCTCAGATGTTGCCATAATGAACCTCATTCAAAC 3984 TGGTGCTGTGGACAGTCTTTCCCTCTCCCTCCCCTTTTAGTTTACGGGAA 4034 TGTTTCCTTTATGGAAAAAAAGTGACTTGTCATTTTGAAGACCCTATATC 4084 TAACATAAGCCTGATTGATGTTCATGGTGTTTTCAACTCCATAAATTGCT 4134 GGGTCTAACCAGTCTCCCTTGAAATCCATGTTTTCCAATAGGAGATAACC 4184 AGGGTGACCACAGCTCTTGGAAGGAAAAGTGGAATTAACTCCAATGTATC 4234 CTGATGAACAACAACAACAAAAATNTGGTACAACTTGCCCTTGAGGCCAA 4284 GCCAAGTCCATAGCATTCACCATGATCATATGCCCCTCGGGATCCTGANA 4334 NAAANANANAGGCTTGTACTGAGGGGTNTTCCCATTGTGGGGGTCGCAGGG 4384 AGGGAAAAAGCCAGGAAGGCAGTGGTCATTNTCCCAAAGTCCACCCATCGT 4434 AAGGAGGTGACAGATCGGAGTCAGCAGATTAGTAATTCAAATGGGTAATG 4484 GAAAAGATTCCTTTAAGCTTCATTTTTCAGAGACCATCACTTTAGAAAAT 4534 CAGAGAAATCCTGTTTTGATACTTNTTAGTTAAAATAATATGTTACCGTT 4584 TATCTGGTACTTCATTTTNTTGACTAAAATTACTTTTCACTTTAAGCTTG 4634 AATAAAAATTTTCATTCATAACTGTAAAAAAAAAAAAAAAAAAAAAAAAA

FIG. 3. Nucleotide sequence of mouse Pfet $1 \mathrm{cDNA}$ and its deduced amino acid sequence. Nucleotide numbers are shown on the left and amino acid sequence numbers are shown in parentheses on the right. The Kozak consensus sequence is outlined in red. The tetramerization domain is underlined. An asterisk denotes the stop codon. Putative polyadenylation consensus sequences are shaded. 


\begin{tabular}{|c|c|c|c|}
\hline onsensus: & 1 & VRLNVGGKRFETSKSTLTRF - PDTRLGRLLKC- - - - - -RDCDFYDDETGEYFFD & 48 \\
\hline hPFET1: & 35 & VELNVGGQVYVTRRCTVVSV - PDSLLWRMFTQ- - - - -QQPQELARDSKGRFFLD & 83 \\
\hline mPFET1: & 35 & IVELNVGGQVYVTRRCTVVSV-PDSLLWRMFTQ- - - - -QQPQELARDSKGRFFLD & 83 \\
\hline gi 10835752 : & 2 & RVVINISGLRFETQLKTLAQF-PETLLGDPKK-------R-MRYFDPLRNEYFFD & 47 \\
\hline $3387822:$ & 82 & RILINVSGQQFEISSELLDRH - PSTLLGNQAS - - - - - R-SKFYDKRKKEYFFD & 127 \\
\hline $24418469:$ & 58 & FVIINVGGIKYKVPWTTLENC-PLTRLGKLKSCNNYDEIMNICDDYDVSCNEFFFD & 112 \\
\hline $688438:$ & 65 & RILLNVGGVRHETHVSTLRTV-PNSRLSRLAET-- - - - - - HLENGGGRQEYFFD & 110 \\
\hline 1763617: & 38 & $\begin{array}{l}\text { ELRLNVSGFMYRLHESFLNRF } \\
* * \star * *{ }_{*}\end{array}$ & 82 \\
\hline
\end{tabular}

consensus: $\quad 49$ RSPKHFEHILNFYRTG-GKLHRPEEVCLES-FLEEAEFYGLDELA--IELCCEDE

hPFET1:

84 RDGFLFRYILDYLRDL--QLVLPDYFPERSRLQREAEYFELPELV--RRLGAPQQ

MPFET1 :

84 RDGFFFRYILDYLRDL--QLVLPDYFPERSRLQREAEYFELPELV--RRLGAPQQ

gi 10835752: 48 RNRPSFDAILYYYQSG-GRLRRPVNVPLDI-FSEEIRFYELGEEA--MEMFREDE

gi 3387822 :

128 RHRPTFEIVFAYFQYG-GKLRRPDYIPDDV-FLNEIEYYQLEPEV - - IEDYKISE

gi 24418469 :

113 RNPSAFRTIMTFLTA--GKLRLLREMCALS-FQEELVYWGIEEDH - -LEWCCKKR

gi 688438:

111 RHPSVFNSIIDFYRT - -RELHVPLEVCGAV-VKRELDFWQINELE- - IKACCWRH

gi 10835752: voltage-gated potassium channel Kv1

gi 3387822: voltage-gated potassium-channel LKv1

gi 24418469: potassium voltage-gated channel subfamily G member 2 cKv6.2

gi 688438: non-inactivating potassium channel

gi 1763617: potassium channel gamma subunit

FIG. 4. Alignment of the consensus sequence for the tetramerization domain of the voltage-gated $\mathrm{K}^{+}$channel family (PFAM00214) (Bixby et al. 1999), deduced amino acid sequence of the tetramerization domain from the human and mouse Pfet 1 genes, and various potassium channel tetramerization domains. Dashes represent gaps

\section{Northern blot analysis}

To determine the relative level of expression of human PFET1 mRNA in various tissues, Northern blot analysis was performed using the original 3 ' cochlear cDNA clone ( $0.9 \mathrm{~kb}$ of $3^{\prime}$ UTR) to probe a panel of human fetal RNA samples (Fig. 5). One transcript $(\sim 6 \mathrm{~kb})$ was revealed at high levels in human fetal cochlea and brain, at moderate levels in skeletal muscle, lung, ovary, and eye, and at lower levels in thymus, tongue, heart, and adrenal gland (Fig. 5A). A Northern blot panel of adult and fetal human organs was hybridized with different PFET1 probes (Fig. 5B, D). In contrast to the abundant fetal expression pattern, the $\sim 6 \mathrm{~kb}$ PFET1 transcript was present at barely detectable levels in adult tissues studied, such as spinal cord, cerebrum, cerebellum, skeletal muscle, lung, and lymph node (Fig. 5B). Low expression levels of the PFET1 transcript were also detected in adult liver, heart, and kidney (data not shown). The transcript was also detected in various regions of the fetal brain (Fig. 5C). Other smaller-sized bands, in particular a $\sim 4.7 \mathrm{~kb}$ band, were identified by probes introduced to align the sequences. Asterisks denote amino acids conserved across four subfamilies of voltage-gated $\mathrm{K}^{+}$channels (Bixby et al. 1999). Red letters represent highly conserved regions across a more diverse group of PFAM00214 family members (only 5 out of 25 were listed) using NCBI's Conserved Domain Database (CDD).

made from the PFET1 ORF (Fig. 5B, ORF probe) and are thought to be due to nonspecific binding since the ORF region is $70 \%$ GC-rich and because the $3^{\prime}$ internal UTR probe, which would be expected to be present in a transcript of this length, does not identify these bands (see Fig. 5B, 3' UTR internal).

For Northern blot analysis of total RNA from adult mouse tissues, we initially used the "ORF probe" containing part of the $3^{\prime}$ ORF region and part of the $3^{\prime}$ UTR. A single $\sim 6 \mathrm{~kb}$ transcript was revealed at low levels in most adult mouse tissues tested (Figs. 6A, D). Then, using a probe to a downstream $3^{\prime}$ UTR region (" 3 ' UTR probe 1 , which overlaps with the "ORF probe"), three transcripts (approximately 4, 4.5, and $6 \mathrm{~kb}$ ) were identified in samples containing aging mouse brain and whole mouse embryos at different embryonic stages (Figs. 6B, C, D). The intensity level of the largest Pfet 1 mouse transcript identified during embryogenesis appears to increase between days 11.5 and 15.5, while the intensity level of the smaller mouse transcripts seems to peak earlier, from days 6 to 15.5 (Fig. 6C). The smaller transcripts are not as apparent in the adult tissue panel, and most likely 
A

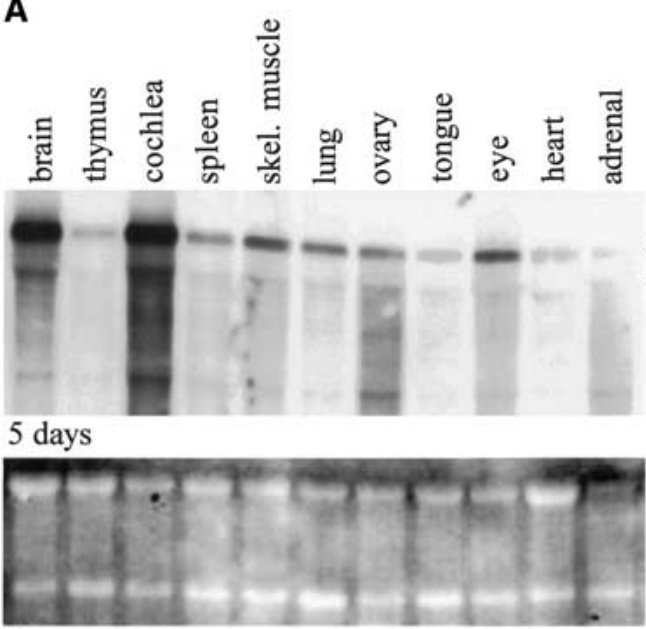

$\mathrm{EtBr}$

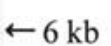

$\leftarrow 4.7 \mathrm{~kb}$

$18 \mathrm{~S}$

C

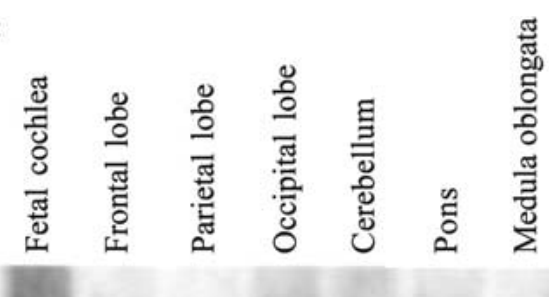

B

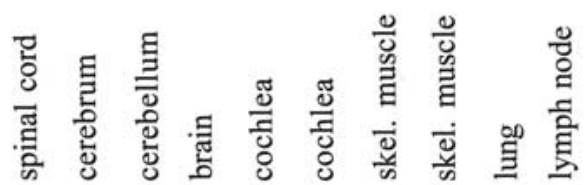

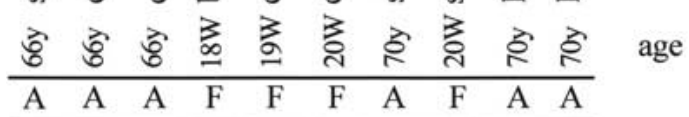

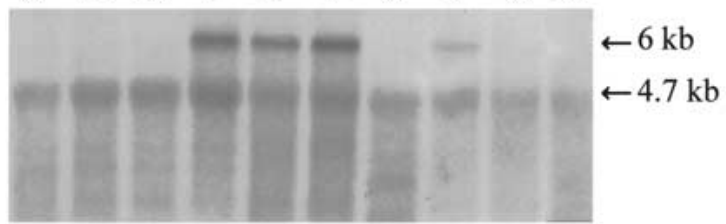

ORF probes $2 \mathrm{wks}$

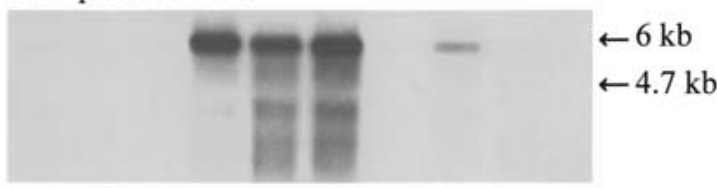

3'UTR internal 5 days

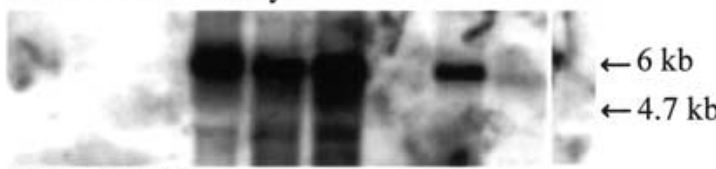

3'UTR MT 5 days

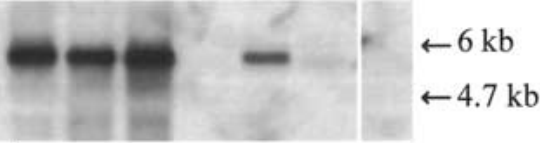

3'UTR MT 1 day

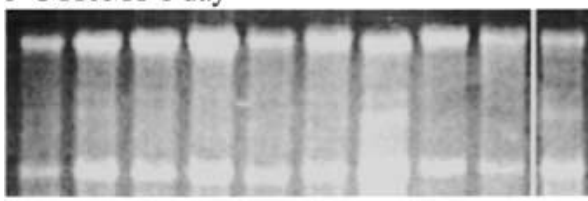

\section{D}

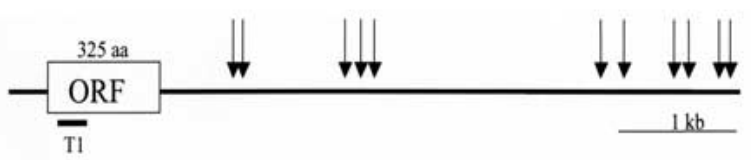

$\begin{array}{ccc}\underbrace{240}_{78 \%} & 978 & 70 \%\end{array}$

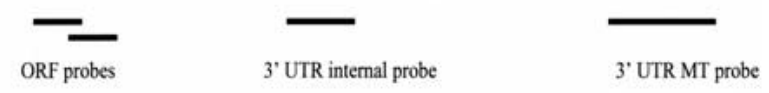


FIG. 6. Northern blot analysis of mouse RNA samples hybridized with mouse Pfet1 radiolabeled fragments. A Adult mouse tissue panel containing $10 \mu \mathrm{g}$ of each sample hybridized with a probe to the 3 end of the ORF reveals low expression levels of a single $\sim 6 \mathrm{~kb}$ transcript in adult mouse tissues. A lane containing $10 \mu \mathrm{g}$ of human fetal brain total RNA is present for comparison. A, adult; $M$, mouse. B Mouse aging brain panel containing $20 \mu \mathrm{g}$ of each sample hybridized with the $3^{\prime}$ UTR probe 1 reveals the presence of a predominant $\sim 6 \mathrm{~kb}$ transcript and two smaller and less intense transcripts ( $\sim 4$ and $4.5 \mathrm{~kb}$ ) in the embryonic and newborn stages. C Mouse developmental panel containing $20 \mu \mathrm{g}$ of each sample hybridized with a probe to the $3^{\prime}$ end of the ORF reveals two transcripts. The upper transcript $(\sim 6 \mathrm{~kb})$ has a higher level of intensity between days 11.5 and 16.5 while the lower transcripts ( $\sim 4$ and $4.5 \mathrm{~kb}$ ) have a higher level of intensity between days 6.5 and 16.5. For each Northern blot, a photograph of the EtBr-stained RNA gel is shown. D Schematic of the mouse Pfet1 gene. The position of the ORF and the polyadenylation consensus sequences (arrows) are shown relative to each other. The position of the tetramerization domain (T1) is indicated. The positions of $5^{\prime}$ and $3^{\prime}$ regions of Pfet 1 that were amplified by PCR or used for probes are indicated. The percent of GC-richness of various regions within the Pfet 1 gene is depicted at the bottom of the diagram.

reflect the lower concentration of RNA used to prepare that Northern blot. Interestingly, the smallest mouse transcript $(\sim 4 \mathrm{~kb})$ was not identified by a probe derived from the $3^{\prime}$-most mouse region $\left(3^{\prime}\right.$ UTR probe 2) (data not shown) suggesting the use of an internal polyadenylation sequence.

Northern blot analysis utilizing probes derived from different regions of the human gene gave the same results as obtained with a $3{ }^{\prime}$-most probe and did not reveal the presence of additional transcripts for either human or mouse genes (data not shown).

FIG. 5. Autoradiographs of Northern blots containing $10 \mu \mathrm{g}$ per lane of total human RNAs hybridized with radiolabeled PFET1 fragments. A Panel of human fetal tissues probed with the 3'-most UTR PFET1 probe (MT fragment) demonstrates expression of a single transcript of $\sim 6 \mathrm{~kb}$ in most tissues, with highest expression in cochlea and brain. B Panel of adult ("A") and fetal ("F") RNAs probed with various PFET1 probes demonstrates high levels of expression of a single $\sim 6 \mathrm{~kb}$ transcript in fetal tissues and barely detectable levels in adult tissues, including spinal cord, cerebrum, cerebellum, skeletal muscle, lung, and lymph node. Similar low-level expression was observed in the other adult tissues that are not shown, including lung, heart, kidney, and liver. C Panel of total RNA isolated from different regions of the fetal brain probed with PFET1; a single $\sim 6 \mathrm{~kb}$ transcript is detected in all samples. A lane of fetal cochlear RNA was included as a positive control. For each Northern blot, a photograph of the EtBr-stained RNA gel is shown. F, fetal; A, adult; y, years; W, weeks. D Schematic of PFET1 gene. The position of the ORF and the polyadenylation consensus sequences (arrows) are shown relative to each other. The position of the tetramerization domain (T1) is indicated. The positions of $5^{\prime}$ and $3^{\prime}$ regions of PFET1 that were amplified by PCR or used for probes are indicated. The "MT" probe is to the 3'-most end of the 3' UTR. The percent GCrichness of various regions within the PFET1 gene is depicted at the bottom of the diagram.
A

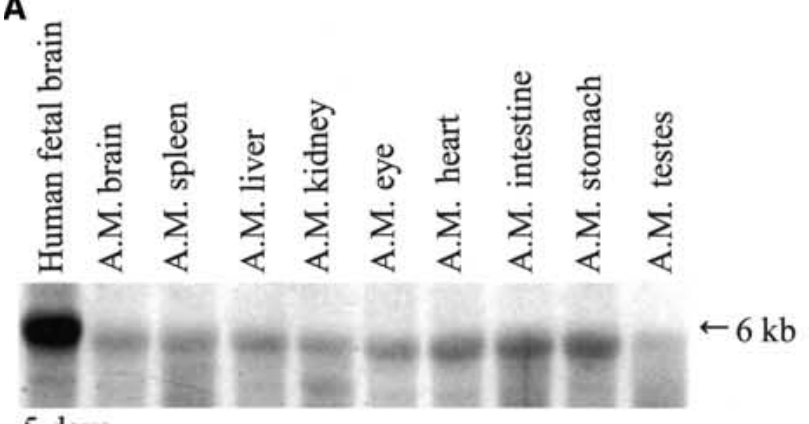

5 days

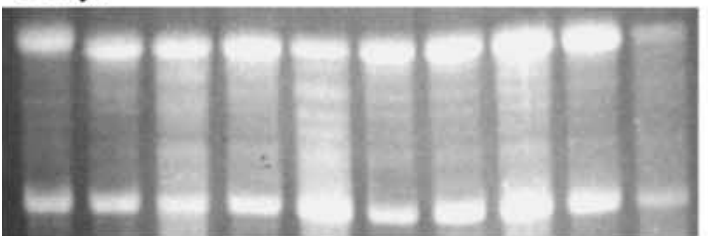

$28 \mathrm{~S}$

$18 \mathrm{~S}$

\section{$\mathrm{EtBr}$}

B

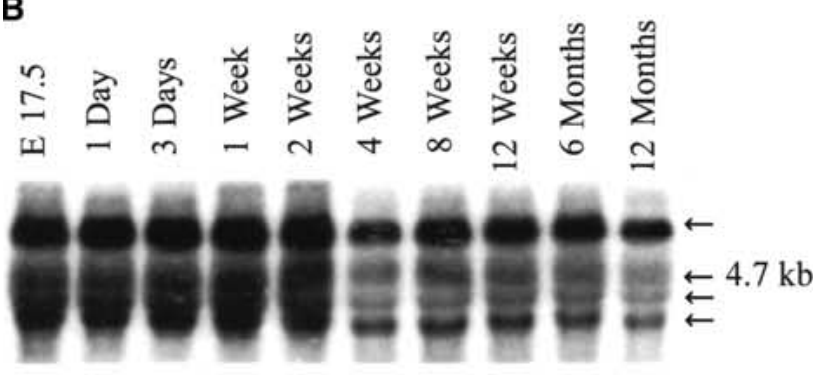

3 days

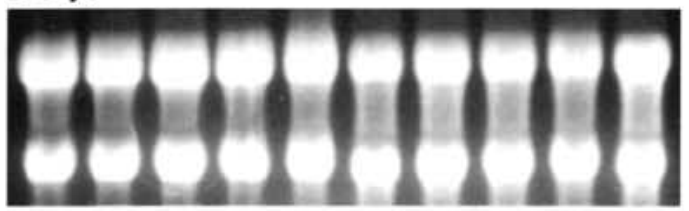

$28 \mathrm{~S}$

$18 \mathrm{~S}$

$\mathrm{EtBr}$

C

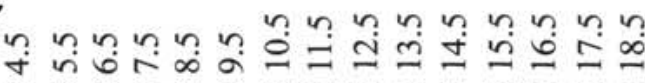

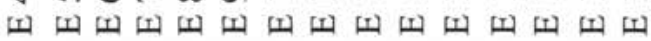

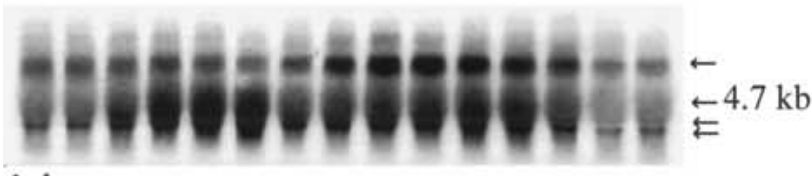
1 day

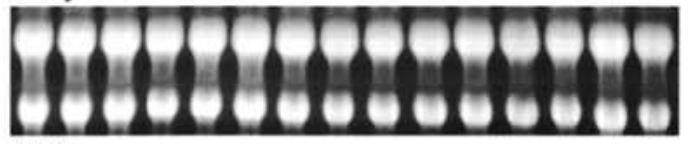

$\mathrm{EtBr}$

D

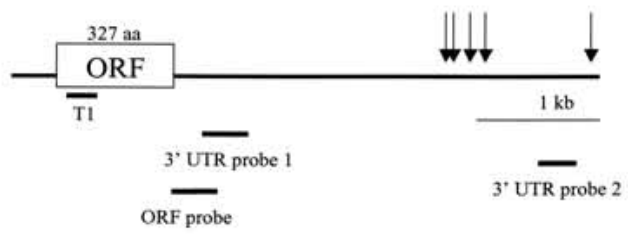




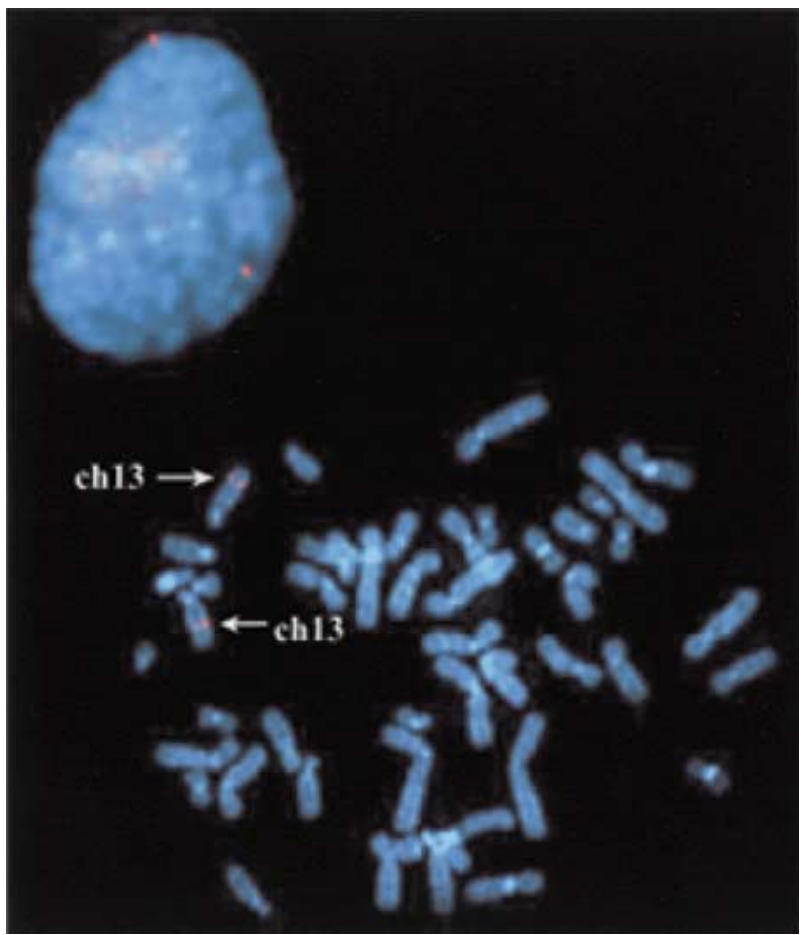

FIG. 7. Chromosomal localization by fluorescence in situ hybridization (FISH) of a human PAC containing the entire PFET1 gene. Human metaphase chromosomes are counterstained with DAPI following FISH with PAC 246 J2. Arrows indicate the positions of the signals that localize to band q21 on both chromosome $13 \mathrm{~s}$.

\section{Chromosomal mapping in human}

Physical mapping of PFET1 was done to determine its chromosomal position and to assess whether it is located within a region of any known deafness loci, making it a positional candidate gene for that deafness disorder. Initially, PFET1 was localized to chromosome 13 by virtue of its sequence homology to a genomic chromosome 13 PAC (GenBank accession No. AC000403, PAC 246 J2) and to several chromosome 13 STSs (SHGC-15652, WI-17550, and TIGRA002 N08). PAC $246 \mathrm{~J} 2$, which contains the entire PFET1 gene, was FISH mapped and localized to band q21 on chromosome 13 (Fig. 7). Currently, PFET1 is not a candidate gene for any known deafness disorder as none has yet been mapped within this chromosomal band (Van Camp and Smith 2003). The mouse Pfet1 gene was mapped to chromosome 14 near the markers D14Mit8, D14Mit93, and D14Mit145.1 as determined by sequence identity with the Celera sequence GA_x5 J8B7 W5Y0C. Human 13q21 is contained within a region of homologous synteny in mouse chromosome 14 and, thus far, no mouse deafness mutant has been identified near the Pfet 1 gene region.
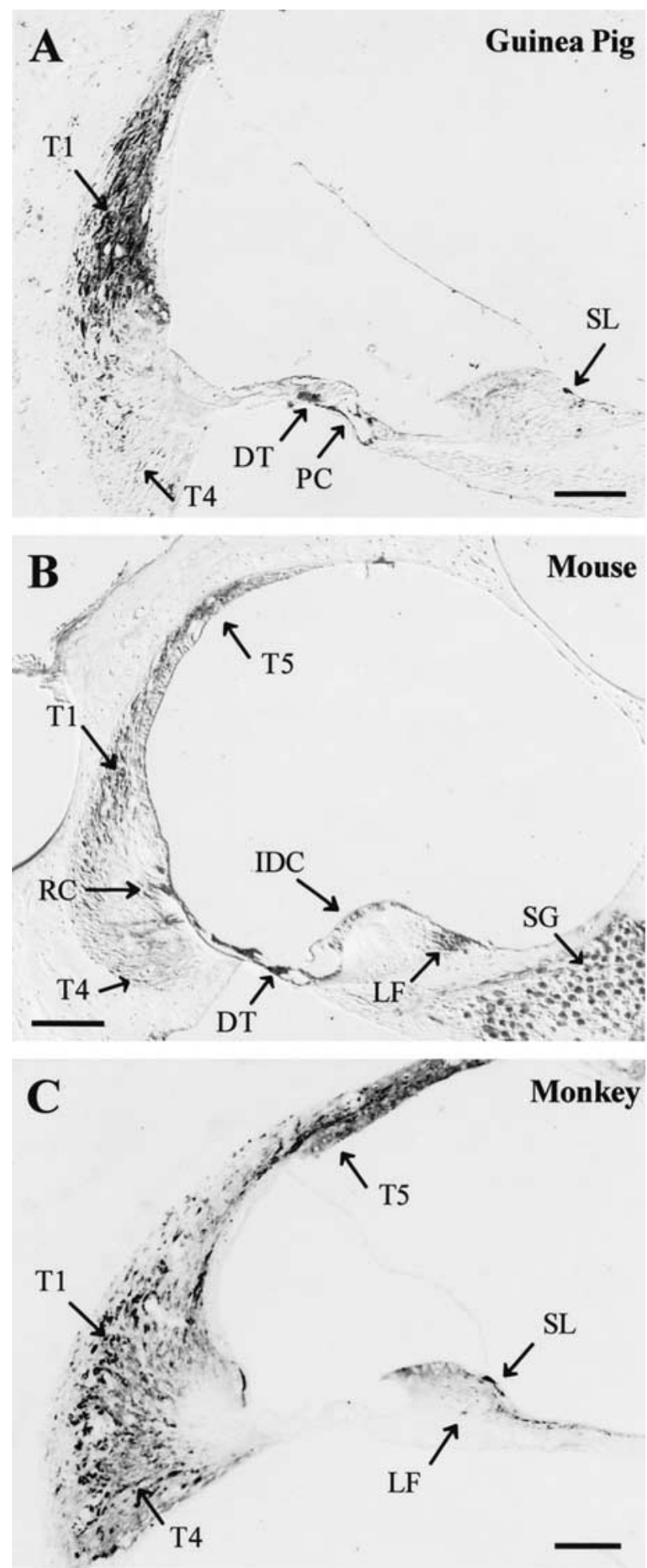

FIG. 8. Immunohistochemical staining using pfetin antibody on (A) formalin glutaraldehyde (FG)-fixed guinea pig, (B) FG-fixed mouse, and (C) FA-fixed monkey cochleas. Arrows point to individual cells positive for immunostaining with pfetin antibody. Positive immunostaining is seen as the dark DAB reaction product. Dominant staining is seen in type I fibrocytes of the cochlea. T1, 4, 5 = types I, $\mathrm{IV}$, and $\mathrm{V}$ fibrocytes; $\mathrm{PC}=$ pillar cell; IDC = interdental cell; ISC = inner sulcus cell; $\mathrm{SL}=$ supralimbal cell; $\mathrm{LF}=$ limbal fibrocytes; $\mathrm{RC}=$ root cells; $\mathrm{SG}=$ spiral ganglion cells. Each scale bar represents $100 \mu \mathrm{m}$. 


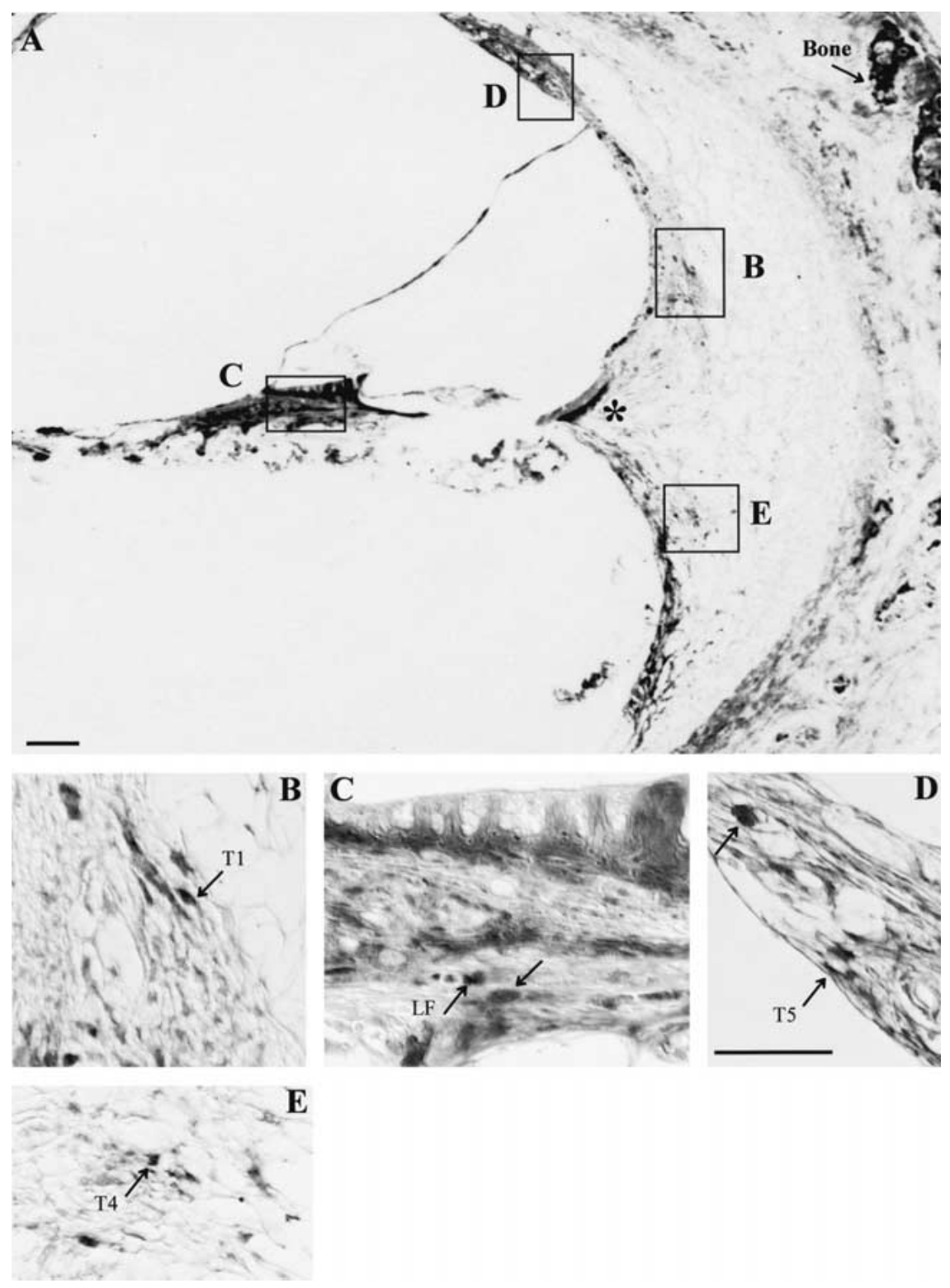

FIG. 9. Immunohistochemical staining using pfetin antibody on formalin-fixed adult human cochlea (A). Higher magnification views of the boxed areas are shown in correspondingly lettered images below (B-E). Arrows point to immunostained cells. Immunostaining is present in type I (T1), IV (T4), and V (T5) fibrocytes. LF = limbal fibrocytes; $*$ extracellular matrix. Each scale bar represents $100 \mu \mathrm{m}$. The images in $\mathbf{B}-\mathbf{E}$ are all the same magnification.

\section{Immunohistochemical analysis}

Before immunostaining was attempted, the antibody was tested for purity and specificity using Western blot analyses. The analyses of purified pfetin antibody on both bacterially expressed proteins and tissues extracted from various mouse organs (6-month-old brain, heart, and skeletal muscle) showed one distinct band between 35 and $47 \mathrm{kDa}$, the expected size of pfetin, for the positive control but not for either of the negative controls (data not shown).

The mammalian cochlear and vestibular systems consist of various cell types. In the cochlea, the greatest number of immunostained cells were type I fibrocytes in the spiral ligament (Fig. 8). This finding was observed in all species, including human, mon- key, mouse, and guinea pig (Figs. 8 and 9B). Immunostaining was also localized in the following cochlear cell classes: types IV and V fibrocytes, Deiters' cells, inner and outer pillar cells, inner sulcus cells, interdental cells, and supralimbal and limbal fibrocytes (Figs. 8 and 9). Type V fibrocytes, also called suprastrial cells, were positive for immunostaining near Reissner's membrane in human (Fig. 9D), monkey (Fig. 8C), guinea pig (Figs. 10C) and most of the mouse cochlear sections (Fig. 8B). While immunostaining of limbal fibrocytes and supralimbal cells was observed in mouse, guinea pig, monkey, and human cochleas (Figs. 8 and 9), immunostaining in Deiters' cells, inner and outer pillar cells, and interdental cells was observed only in mouse and guinea pig (Fig. 10A-C). Immunostained 

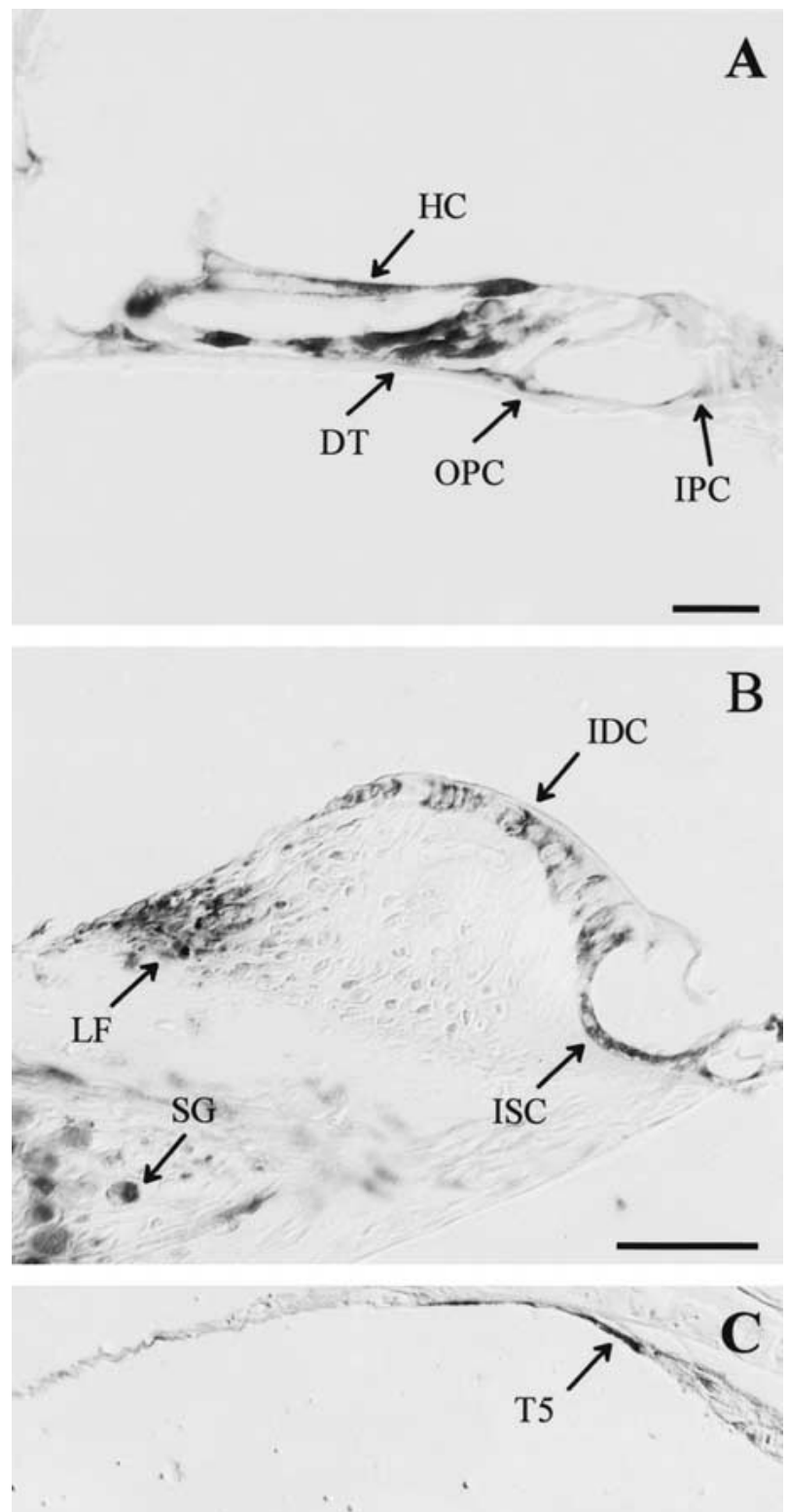

RM

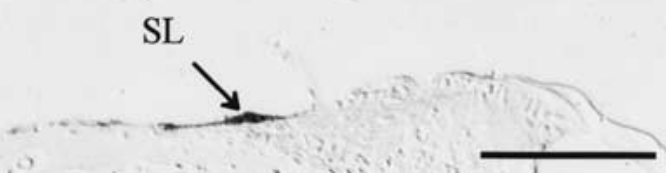

FIG. 10. Immunostained formalin plus glutaraldehyde-fixed mouse $(\mathbf{A}, \mathbf{B})$ and formalin acetic acid-fixed guinea pig $(\mathbf{C})$ cochlea. A Organ of corti, B the spiral limbus, and C areas contacting Reissner's membrane. IPC, OPC = inner and outer pillar cells; DT $=$ Deiters' cells; ISC = inner sulcus cells; IDC = interdental cells; LF = limbral fibrocytes; $\mathrm{HC}=$ Hensen cells; $\mathrm{SG}=$ spiral ganglion; $\mathrm{T} 5$ = type $\mathrm{V}$ fibrocytes (suprastrial cells); $\mathrm{SL}=$ supralimbal cells. Each scale bar represents $50 \mu \mathrm{m}$. neurons included spiral ganglion cells (Fig. 8B), Scarpa's ganglion cells, and Purkinje cells in mouse sections containing brain tissues (not shown).

In the vestibular system apical immunostaining of type I hair cells was detected at the cuticular plate and in the hair cell cytoplasm (Figs. 11G,H). Beneath the sensory epithelium, connective tissue cells were also immunostained in formalin plus glutaraldehydefixed mouse (Fig. 11H) and guinea pig ampulla (Figs. 11E,F,H). Positively stained fibrocytes extended from beneath the sensory epithelium to the area beneath the vestibular dark cells. Adjacent to the sensory epithelium, transitional cells and cells beneath the vestibular dark cells in human fetal and guinea pig (Figs. 11A,B,E,F) were positive. In addition, positive cells were present facing the lumen of the semicircular canals in human fetal tissue (Figs. 11C,D) and along with the lumenal and ablumenal surface of formalin plus glutaraldehyde-fixed mouse and guinea pig semicircular canal (Figs. $11 \mathrm{E}, \mathrm{F}, \mathrm{H})$.

These immunohistochemical analyses were performed mostly on adult tissues, because fetal tissues were not available at the time of this study. Although pfetin has a low expression in adult tissues, nonetheless it is expressed in a variety of adult organs and therefore the immunohistochemical analyses on adult organs remains a reasonable initial study to carry out. Immunohistochemical analyses on fetal organs are pertinent and such future analyses will be performed.

\section{DISCUSSION}

We have cloned and characterized a novel intronless human gene designated PFET1 and its mouse homolog, Pfet1. The ORFs of PFET1 and Pfet 1 are unusually GC-rich $(70 \%)$; the potential significance of this is unknown. The encoded proteins of both genes contain a tetramerization domain characteristic of voltage-gated potassium channel subunits. The $3^{\prime}$ untranslated region from the human gene is long (4996 bp) as the average $3^{\prime}$ UTR length for human mRNAs deposited in public databases is between 740 and 755 base pairs (Pesole et al. 1997, 2000). The role of the unusually long $3^{\prime}$ UTR in this gene is presently unknown. Although 12 putative polyadenylation consensus sequences are predicted in the human sequence, the finding that $5^{\prime}$ and $3^{\prime}$ probes identified only the largest transcript suggests that the other 11 putative polyadenylation consensus sequences may not be utilized to produce alternatively sized transcripts. It remains possible that other PFET1 transcripts of different sizes exist and are at levels below the limits detectable by Northern blot analysis or are 


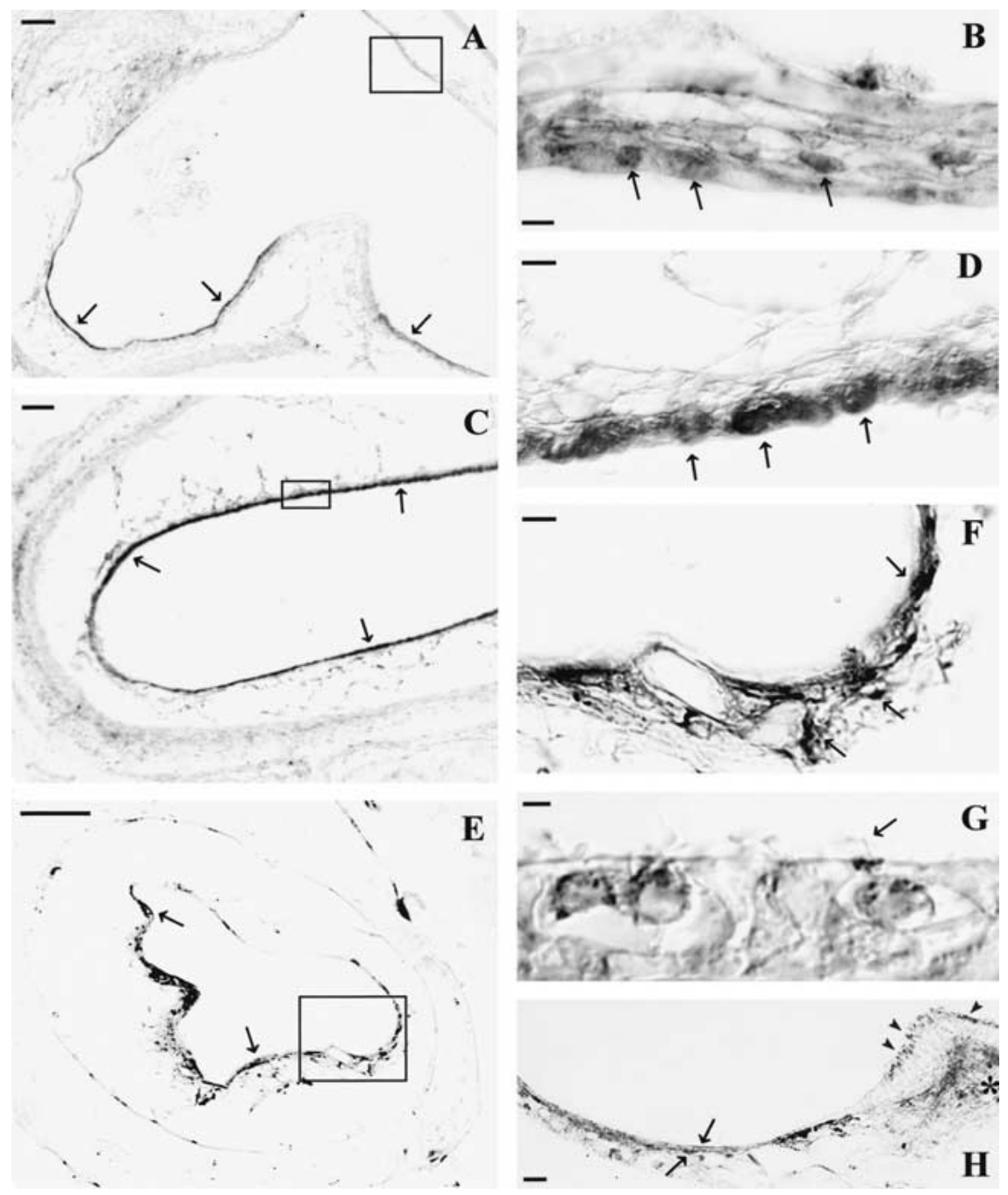

FIG. 11. Pfetin immunostaining of vestibular tissue of 20-week-old human fetus (formalin-fixed) (A-D), guinea pig (formalin plus glutaraldehyde-fixed) $(\mathbf{E}, \mathbf{F})$, and mouse (formalin plus glutaraldehyde-fixed) $(\mathbf{G}, \mathbf{H})$. The semicircular canal shows $(\mathbf{A})$ a continuous layer of staining (arrow) in the lateral ampulla underlying the transitional cells and vestibular dark cells. B Higher magnification of boxed region in A. Arrows point to individual positive cells. C Arrows indicate immunostained cells lining the lumen of the semicircular canal. D Higher magnification of boxed region in C. E Guinea pig posterior ampulla with arrows pointing to immunostained cells. F Higher magnification of boxed region in E. G Sensory epithelium of the macula of the saccule showing the immunostained type I hair cells. Arrow points to the unstained ciliary bundle extending from the darkly stained cuticular plate. The adjacent type I hair cells also show granular reaction products within their cytosol. $\mathbf{H}$ Mouse lateral ampulla with arrows pointing to immunostained lumenal and ablumenal cells. Arrowheads point to cuticular plate staining of type I hair cells. Asterisk indicates staining in the connective fibrocytes underneath the sensory epithelium. Scale bars in A, C, and $\mathbf{E}$ represent $100 \mu \mathrm{m}$; in $\mathbf{B}, \mathbf{D}, \mathbf{F}$, and $\mathbf{G}, 10 \mu \mathrm{m}$; in $\mathbf{H}, 20 \mu \mathrm{m}$. expressed in a different temporal or spatial fashion not tested.

PFET1 encodes a single $\sim 6 \mathrm{~kb}$ transcript and its mouse homolog encodes three transcripts (4, 4.5, and $6 \mathrm{~kb}$ ). In humans, a $6 \mathrm{~kb}$ PFET1 transcript is expressed abundantly in a variety of tissues in the fetus and at strikingly lower levels in the adult. The observation that PFET1 is expressed at much higher levels in fetal organs than in adult organs is intriguing. This expression difference in adult and fetal tissue samples appears also to occur with the mouse Pfet 1 transcripts in brain (compare Fig. 6A and B). The disparate expression levels of the PFET1 $6 \mathrm{~kb}$ transcript cannot be explained by the expression of tissue-specific or age-specific alternative transcripts as both $5^{\prime}$ (containing part of the ORF) and $3^{\prime}$ UTR probes identified only a single transcript, the $\sim 6 \mathrm{~kb}$ transcript, in all fetal and adult human tissues tested (see Fig. 5D for position of probes). PFET1 is the first example to our knowledge of a human cochlear gene with such disparate expression patterns in adult and fetal human organs as late as second-trimester devel- opmental age in humans. A similar type of expression has been seen before in other species such as mouse, zebrafish, Xenopus, and chick with genes like GATA3, Pax2, Bmp4, and Bmp7. This type of expression pattern suggests that PFET1 has a developmental role and thus is required at high levels during fetal life and at much lower levels in adulthood. Of note, the human fetal cochlear library from which PFET1 was identified represents largely developmental ages of 16-22 weeks, consistent with cochlea that are morphologically adultlike in structure. There is also evidence that the human fetus responds to sound at about this age. In light of these observations, it is interesting that there is a marked difference in expression levels of PFET1 in the fetus versus the adult, suggesting a potential important role of PFET1 in the cochlea during later stages of fetal life.

\section{Tetramerization domain}

Voltage-gated potassium channels, of which there are multiple families, each consisting of numerous 
members, are assembled as homomeric and heteromeric tetramers from membrane-integrated $\alpha$ subunits; the Shaker-related potassium channel also coassembles with cytosolic $\beta$ subunits (Jan and Jan 1997; Pongs et al. 1999). The assembly of different subunits to form functional heteromeric tetramers is thought to be determined by the amino acid composition of the tetramerization (T1) domain, and thus contributes to the diversity of electrical responses that a cell can generate in response to changes in membrane potential (Bixby et al. 1999). Therefore, it is not surprising to observe some sequence variations in the conserved regions within $\mathrm{K}^{+}$ channel tetramerization domains among family members given the existence of a $\mathrm{K}^{+}$channel tetramerization domain consensus sequence (Fig. 3). The exact role of the $\mathrm{T} 1$ domain remains controversial. Previously, it was shown that the T1 domain is not necessary for $\mathrm{K}^{+}$channel assembly or function (Kobertz and Miller 1999). Rather, the T1 domain of voltage-gated potassium channel subunits may act more as a segregation domain in that it ensures that tetramerization occurs only among subunits belonging to the same family and that cross-family subunit assembly does not occur (Li et al. 1992; Shen and Pfaffinger 1995). The T1 domain may also function as a docking station for the $\beta$ subunit of voltage-activated potassium $(\mathrm{Kv})$ channels such that the removal of the T1 domain disrupts $\beta$ subunit association (Sewing et al. 1996; Gulbis et al. 2000).

Because the predicted ORF of PFET1 contains a tetramerization domain, it is tempting to speculate that pfetin may be a novel voltage-gated $\mathrm{K}^{+}$channel subunit that could contribute to tetramer diversity and thus could participate in a variety of electrical responses of the cell. Furthermore, the deduced amino acid sequence of pfetin is predicted to contain four hydrophobic regions of at least 10-15 amino acids in length. However, since PFET1 is predicted to contain no transmembrane domains, and six are characteristic of voltage-gated potassium channel subunits, it is unlikely that PFET1 encodes another member of the voltage-gated potassium channel subunits.

\section{Relationship between T1 and POZ domains}

The voltage-gated potassium channel tetramerization domain is thought to have a structural and evolutionary relationship to the $\mathrm{BTB} / \mathrm{POZ}$ (for bric-a-brac, tramtrack, broad complex poxvirus and zinc finger) domain, which is found in a variety of proteins involved in transcriptional regulation, cytoskeletal organization, and development (Aravind and Koonin 1999). The POZ domains of the mammalian tran- scriptional represser proteins BCL6 and PLZF (promyelocytic leukemia zinc finger) interact with the transcriptional corepressor proteins mSIN3A and SMRT (silencing mediator of retinoid and thyroid hormone receptor) via a paired amphipathic helix 1 (PAH1) domain (David et al. 1998) and multiple SMRT contacts (Hong et al. 1997), respectively. Chromosomal translocations involved in human leukemias generate fusion proteins, such as RARAPLZF, containing POZ domains that play an important role in the pathology of the disease (Hong et al. 1997; Grignani et al. 1998; Lin et al. 1998). The BTB/ POZ domain, like the voltage-gated potassium channel T1 domain, is important for protein-protein interactions and allows for dimerization of $\mathrm{BTB} / \mathrm{POZ}$ domain-containing proteins. However, unlike the voltage-gated potassium channel $\mathrm{T} 1$ domain, POZ domains mediate interaction between proteins containing other domains as well. Future studies are necessary to determine if the $\mathrm{T} 1$ domain present in PFET1/Pfet1 is functional and possibly acts like a $\mathrm{BTB} / \mathrm{POZ}$ domain, allowing for the interaction of PFET1/Pfet1 with proteins containing various types of domains.

\section{Immunohistochemistry of pfetin antibody}

The most numerous cochlear cells positive for pfetin were type I fibrocytes of the spiral ligament in the cochlea. Fibrocytes of the ligament are thought to be part of the connective tissue cell gap junction system (Kikuchi et al. 2000) and may play a role in $\mathrm{K}^{+}$recycling by transporting $\mathrm{K}^{+}$to the stria vascularis (Spicer and Schulte 1997). The loss of type I fibrocytes of the spiral ligament is the predominant histopathology of DFNA9 (Merchant et al. 2000), a known autosomal dominant, nonsyndromic, progressive sensorineural hearing loss (Robertson et al. 1998). These are also the cells that are most severely disrupted in the hydropic guinea pig (Ichimiya et al. 1994). The type I fibrocytes, containing enzymes such as intracellular $\mathrm{Ca}^{2+}-$ ATPase, carbonic anhydrase, aldehyde dehydrogenase, and calcium-binding proteins, are thought to be involved in the regulation of cochlear fluid and ion balance (Ichimiya et al. 1994; Spicer et al. 1997). Although the exact function of type I fibrocytes in cochlea has not been clearly defined, it is quite obvious that their loss in DFNA9 shows that they play an essential role in normal auditory functions. Besides type I fibrocytes, pfetin antibody also stains type IV and type $\mathrm{V}$ fibrocyte cells in the cochlea across different species. Pfetin antibody immunostaining in limbal fibrocytes, supralimbal, Deiters', interdental, and pillar cells was less consistent. One possible explanation is species-specific expression of pfetin function in the cochlea. The amount of antigen 
available for pfetin antibody binding might vary somewhat as well depending on the plane of the section giving certain cellular structure a better exposure. However, the quality of tissue fixation might also have contributed to some of the staining variation. Initial attempts at immunostaining formalin-mixed mouse cochlea were negative. Experimentation with other fixatives revealed consistent staining patterns with tissues fixed in formalin plus glutaraldehyde. Although human material that was promptly fixed with formalin plus glutaraldehyde was not available, it seems likely that the immunostaining results from the human sections is credible because of its similarity to the patterns of immunostaining seen in animal tissue where control of fixation was possible. It remains to be confirmed that vestibular hair cells and fibrocytes are pfetin positive in human material.

It is striking that most of the cell classes stained by pfetin antibody in the cochlea have been implicated in the potassium recycling pathway through putative lateral uptake by Deiters' cells, forward through supporting cells, outer sulcus cells, and spiral ligament fibrocytes, and on to strial marginal cells. Given the cell types stained by pfetin antibody and their associated function in the cochlea, it is possible that pfetin plays a role in ion transport or ionic content regulation in the cochlea. This hypothesis is especially intriguing given the presence of the it $\mathrm{T} 1$ domain in pfetin, since this domain is characteristic of $\mathrm{K}^{+}$channel subunits.

An exciting discovery came from the vestibular sensory epithelium where the cuticular plate and the cytoplasm of the type I hair cells were immunostained. Although the function of this gene product is not yet clear, loss of gene function would likely result in a vestibular phenotype if pfetin plays a key role in hair cell function. Similar to the type I fibrocytes stained in the cochlea, connective tissue cells in the vestibular system show pfetin immunostaining as well. Specifically, immunostaining for pfetin in the human fetal, mouse, and some guinea pig vestibular sections reveals a positive signal in a layer of cells underlying the transitional cells and vestibular dark cells. In mouse sections, the connective fibrocytes underneath the vestibular sensory epithelium are prominently stained by pfetin antibody. The functions of these cells are not known. In addition, little is known about the pfetin-positive cell layer that lines the lumen of semicircular canals. They form the barrier that faces the endolymphatic space. It is possible that they play some role in ionic content regulation in the vestibule. Because $\mathrm{T} 1$ domains are members of the POZ domain superfamily, and some proteins containing these domains are involved in cytoskeletal organization, pfetin may also function, by protein-protein interaction via the T1 domain, in the structural or- ganization of the cochlea and vestibule. Given the presence of pfetin in a variety of cell classes such as sensory cells, nerve cells, epithelial cells, and connective tissue cells in the cochlea and vestibular system, it is likely that pfetin could have broad functional roles in the inner ear and the vestibular system. Additional studies are needed to further elucidate the function of this intriguing novel gene.

\section{ACKNOWLEDGMENTS}

We thank Steve Herrick for help with preparation of chromosome spreads and Dr. Charles Lee for assistance with the fluorescence in situ hybridization and chromosome analysis. We thank Robert Blaustein for critical reading of the manuscript. This work was supported by the NIH Grants DC03402 (CCM), F32DC00405 (BLR), T32DC0019 (SFK), and DC03929 (JCA).

\section{REFERENCES}

ADAms JC. Biotin amplification of biotin and horseradish peroxidase signals in histochemical stains. J. Histochem. Cytochem. 40:1457-1463, 1992.

ARAVIND L, Koonin EV. Fold prediction and evolutionary analysis of the POZ domain: structural and evolutionary relationship with the potassium channel tetramerization domain. J. Mol. Biol. 285:1353-1361, 1999.

Bateman A, Birney E, Durbin R, Eddy SR, Howe KL, Sonnhammer EL. The Pfam protein families database. Nucleic Acids Res. 28:263-266, 2000.

Bixby KA, Nanao MH, Shen NV, Kreusch A, Bellamy H, Pfaffinger S, ChoE S. $\mathrm{Zn}^{2+}$ binding and molecular determinants of tetramerization in voltage-gated $\mathrm{K}^{+}$channels. Nat. Struct. Biol. 6:38-43, 1999.

Chirghin JM, Przybyla AE, MacDonald RJ, Rutter WJ. Isolation of biologically active ribonucleic acid from sources enriched in ribonuclease. Biochemistry 18:5294-5299, 1979.

Cohen-Salmon M, El-Amraoui A, Leibovici M, Petit C. Otogelin: a glycoprotein specific to the acellular membranes of the inner ear. Proc. Natl. Acad. Sci. U.S.A. 94:14450-14455, 1997.

David G, Alland L, Hong SH, Wong CW, DePinho RA, Dejean A. Histone deacetylase associated with $\mathrm{mSin} 3 \mathrm{~A}$ mediates repression by the acute promyelocytic leukemia-associated PLZF protein. Oncogene 16:2549-2556, 1998.

Devereux J, Haeberli P, Smithies O. A comprehensive set of sequence analysis programs for the VAX. Nucleic Acids Res. 12:387-395, 1984.

Feinberg AP, Vogelstein B. A technique for radiolabeling DNA restriction endonuclease fragments to high specific activity [addendum]. Anal. Biochem. 137:266-267, 1984.

Gorlin RJ, Toriello HV, Cohen MM. Hereditary hearing loss and its syndromes. Oxford University Press, Oxford, 1995.

Grignani F, De Matteis S, Nervi C, Tomassoni L, Gelmetti V, Cioce M, Fanelli M, Ruthardt M, Ferrara FF, Zamir I, Seiser C, Lazar S, Minucci S, Pelicci PG. Fusion proteins of the retinoic acid receptor-alpha recruit histone deacetylase in promyelocytic leukaemia. Nature 391:815-818, 1998.

Gulbis JM, Zhou M, Mann S, MacKinnon R. Structure of the cytoplasmic beta subunit-T1 assembly of voltage-dependent $\mathrm{K}^{+}$ channels. Science 289:123-127, 2000. 
Gurish MF, Bell AF, Smith TJ, Ducharme LA, Wang RK, Weis JH. Expression of murine beta 7 , alpha 4 , and beta 1 integrin genes by rodent mast cells. J. Immunol. 149:1964-1972, 1992.

Hedrick SM, Cohen DI, Nielsen EA, Davis MM. Isolation of cDNA clones encoding $\mathrm{T}$ cell-specific membrane-associated proteins. Nature 308:149-153, 1984.

Heller S, Sheane CA, Javed Z, Hudspeth AJ. Molecular markers for cell types of the inner ear and candidate genes for hearing disorders. Proc. Natl. Acad. Sci. U.S.A 95:11400-11405, 1998.

Hoffmann K, Stoffel W. TMbase-A database of membrane spanning proteins segments. Biol. Chem. Hoppe Seyler 347:166, 1993.

Hong SH, David G, Wong CW, Dejean A, Privalsky ML. SMRT corepressor interacts with PLZF and with the PML-retinoic acid receptor alpha (RARalpha) and PLZF-RARalpha oncoproteins associated with acute promyelocytic leukemia. Proc. Natl. Acad. Sci. U.S.A. 94:9028-9033, 1997.

ICHIMIYA S, ADAMS JC, KIMURA RS. Changes in immunostaining of cochleas with experimentally induced endolymphatic hydrops. Ann. Otol. Rhinol. Laryngol. 103:457-468, 1994.

Imamura S, Adams JC. Immunolocalization of peptide 19 and other calcium-binding proteins in the guinea pig cochlea. Anat. Embryol. (Berl.) 194:407-418, 1996.

Jacob AN, Manjunath NA, Bray-Ward P, Kandpal RP. Molecular cloning of a zinc finger gene eZNF from a human inner ear cDNA library, and in situ expression pattern of its mouse homologue in mouse inner ear. Somat. Cell. Mol. Genet. 24:121-129, 1998.

JAN LY, JAN YN. Cloned potassium channels from eukaryotes and prokaryotes. Annu. Rev. Neurosci. 20:91-123, 1997.

JoNes DT, REED RR. Golf: an olfactory neuron-specific G protein involved in odorant signal transduction. Science 244:790-795, 1989.

Kikuchi T, Adams JC, Miyabe Y, So E, Kobayashi T. Potassium ion recycling pathway via gap junction systems in the mammalian cochlea and its interruption in hereditary nonsyndromic deafness. Med. Electron Microsc. 33:51-56, 2000.

Kobertz WR, Miller C. $\mathrm{K}^{+}$channels lacking the 'tetramerization' domain: implications for pore structure. Nat. Struct. Biol. 6:1122-1125, 1999.

Li M, JAN YN, JAN LY. Specification of subunit assembly by the hydrophilic amino-terminal domain of the Shaker potassium channel. Science 257:1225-1230, 1992.

Lin RJ, Nagy L, Inoue S, Shao W, Miller WH Jr, Evans RM. Role of the histone deacetylase complex in acute promyelocytic leukaemia. Nature 391:811-814, 1998.

Merchant SN, Linthicum FH, Nadol JB Jr. Histopathology of the inner ear in DFNA9. Adv. Otorhinolaryngol. 56:212-217, 2000.

Morton NE. Genetic epidemiology of hearing impairment. Ann. N.Y. Acad. Sci. 630:16-31, 1991.

Ohara O, Nagase T, Ishikawa K, Nakajima D, Ohira M, Seki N, Nomura N. Construction and characterization of human brain cDNA libraries suitable for analysis of cDNA clones encoding relatively large proteins. DNA Res. 4:53-59, 1997.

Pesole G, Liuni S, Grillo G, Saccone C. Structural and compositional features of untranslated regions of eukaryotic mRNAs. Gene 205:95-102, 1997.

Pesole G, Grillo G, Larizza A, Liuni S. The untranslated regions of eukaryotic mRNAs: structure, function, evolution and bioinformatic tools for their analysis. Brief Bioinform 1:236-249, 2000 .
Pongs O, Leicher T, Berger M, Roeper J, Bahring R, Wray D, Giese AJ, Silva AJ, Storm JF. Functional and molecular aspects of voltage-gated $\mathrm{K}^{+}$channel beta subunits. Ann. N.Y. Acad. Sci. 868:344-355, 1999.

Resendes BL, Robertson NG, Szustakowski JD, Resendes RJ, Weng CC, Morton CC. Gene discovery in the auditory system: characterization of additional cochlear-expressed sequences. J. Assoc. Res. Otolaryngol. 3:45-53, 2002.

Robertson NG, Khetarpal U, Gutierrez-Espeleta GA, Bieber FR, MorTOn CC. Isolation of novel and known genes from a human fetal cochlear cDNA library using subtractive hybridization and differential screening. Genomics 23:42-50, 1994.

Robertson NG, Lu L, Heller S, Merchant SN, Eavey RD, McKenna JB, Nadol JB Jr, Miyamoto RT, Linthicum FH Jr, Lubianca Neto AJ, Hudspeth AJ, Seidman CE, Morton CC, Seidman JG. Mutations in a novel cochlear gene cause DFNA9, a human nonsyndromic deafness with vestibular dysfunction. Nat. Genet. 20:299-303, 1998.

Robertson NG, Heller S, Lin JS, Resendes BL, Weremowicz S, Denis CS, Bell AM, Hudspeth AJ, Morton CC. A novel conserved cochlear gene, OTOR: identification, expression analysis, and chromosomal mapping. Genomics 66:242-248, 2000.

Sewing S, Roeper J, Pongs O. Kv beta 1 subunit binding specific for shaker-related potassium channel alpha subunits. Neuron 16:455-463, 1996.

Shen NV, Pfaffinger PJ. Molecular recognition and assembly sequences involved in the subfamily-specific assembly of voltagegated $\mathrm{K}^{+}$channel subunit proteins. Neuron 14:625-633, 1995.

Skvorak AB, Robertson NG, Yin Y, Weremowicz S, Her H, Bieber KW, Beisel KW, Lynch ED, Beier DR, Morton CC. An ancient conserved gene expressed in the human inner ear: identification, expression analysis, and chromosomal mapping of human and mouse antiquitin (ATQ1). Genomics 46:191-199, 1997.

Skvorak AB, Weng Z, Yee AJ, Robertson NG, Morton CC. Human cochlear expressed sequence tags provide insight into cochlear gene expression and identify candidate genes for deafness. Hum. Mol. Genet. 8:439-452, 1999.

Sonnhammer EL, von Heijne G, Krogh A. A hidden Markov model for predicting transmembrane helices in protein sequences. Proc. Int. Conf. Intell. Syst. Mol. Biol. 6:175-182, 1998.

Soto-Prior A, Lavigne-Rebillard M, Lenoir M, Ripoll C, RebilLard G, Vago P, Pujol R, Hamel CP. Identification of preferentially expressed cochlear genes by systematic sequencing of a rat cochlea cDNA library. Brain Res. Mol. Brain Res. 47:1-10, 1997.

SPICER SS, SCHUlte BA. Golgi-canalicular reticulum system in ion transporting fibrocytes and outer sulcus epithelium of gerbil cochlea. Anat. Rec. 249:117-127, 1997.

Sicer SS, Gratton MA, Schulte BA. Expression patterns of ion transport enzymes in spiral ligament fibrocytes change in relation to strial atrophy in the aged gerbil cochlea. Hear. Res. 111:93-102, 1997.

Thомаs PS. Hybridization of denatured RNA and small DNA fragments transferred to nitrocellulose. Proc. Natl. Acad. Sci. 77: 5210-5205, 1980.

Tusnady GE, Simon I. Principles governing amino acid composition of integral membrane proteins: application to topology prediction. J. Mol. Biol. 283:489-506, 1998.

VAn CAMP G, Smith RJH. Hereditary hearing loss homepage. http://www.uia.ac.be/dnalab/hhh/, (2003). 